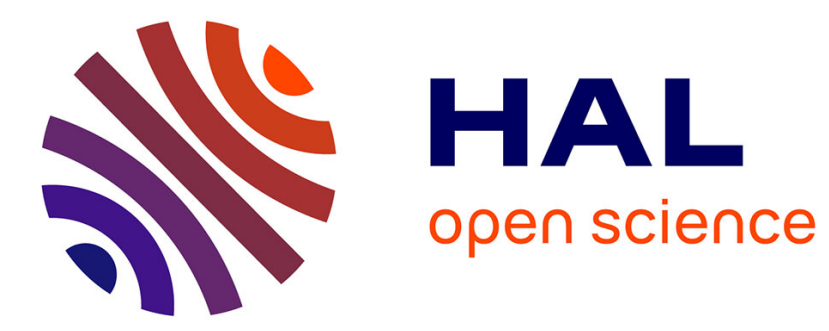

\title{
Controllability with positivity constraints of the Lotka-McKendrick system
}

Nicolas Hegoburu, Pierre Magal, Marius Tucsnak

\section{To cite this version:}

Nicolas Hegoburu, Pierre Magal, Marius Tucsnak. Controllability with positivity constraints of the Lotka-McKendrick system. 2017. hal-01395712v2

\section{HAL Id: hal-01395712 \\ https://hal.science/hal-01395712v2}

Preprint submitted on 30 Aug 2017

HAL is a multi-disciplinary open access archive for the deposit and dissemination of scientific research documents, whether they are published or not. The documents may come from teaching and research institutions in France or abroad, or from public or private research centers.
L'archive ouverte pluridisciplinaire HAL, est destinée au dépôt et à la diffusion de documents scientifiques de niveau recherche, publiés ou non, émanant des établissements d'enseignement et de recherche français ou étrangers, des laboratoires publics ou privés. 


\title{
Controllability with positivity constraints of the Lotka-McKendrick system
}

\author{
Nicolas Hegoburu*, Pierre Magal’, Marius Tucsnak ${ }^{\ddagger}$
}

August 30, 2017

\begin{abstract}
This work considers the linear Lotka-McKendrick system from population dynamics with control active on individuals in a prescribed age range. The main results assert that given $\tau$ large enough (but possibly smaller than the life expectancy), there exists controls driving the system to any equilibrium state or any uncontrolled trajectory in time $\tau$. Moreover, we show that if the initial and final states are positive then the constructed controls preserve the positivity of the population density on the whole time interval $[0, \tau]$. The method is a direct one, in the spirit of some early works on the controllability of hyperbolic systems in one space dimension. Finally, we apply our method to a nonlinear infection-age model.
\end{abstract}

\section{Introduction}

The purpose of this work is the study of the controllability properties of an infinite dimensional dynamical system issued of population dynamics. More precisely, we revisit from a control theoretical view point the linear Lotka-McKendrick model. We aim, in particular, answer to some open questions raised in Barbu, Iannelli and Martcheva [8]. More precisely, let $p(t, a)$ be the distribution of individuals of age $a \geqslant 0$ at time $t \geqslant 0$. Let $a_{\dagger}$ be the life expectancy of an individual and $\tau$ be a positive constant. Let $\beta(a) \geqslant 0$ be the natural fertility-rate and $\mu(a) \geqslant 0$ the natural death-rate of individuals of age $a$. The system we consider, already studied from a control theoretic view point in [8], is described by the equations

$$
\left\{\begin{aligned}
\frac{\partial p}{\partial t}(t, a) & +\frac{\partial p}{\partial a}(t, a)+\mu(a) p(t, a) & & \\
& =m(a) u(t, a), & & (t, a) \in Q_{\tau}, \\
p(t, 0) & =\int_{0}^{a_{\dagger}} \beta(a) p(t, a) \mathrm{d} a, & & t \in(0, \tau), \\
p(0, a) & =p_{0}(a), & & a \in\left(0, a_{\dagger}\right),
\end{aligned}\right.
$$

where $u$ is a control function, $m=\mathbb{1}_{\left[0, a_{0}\right]}$ is the characteristic function of the interval $\left(0, a_{0}\right)$ (where $\left.0<a_{0}<a_{\dagger}\right), p_{0}$ is the initial population density and $Q_{\tau}=(0, \tau) \times\left(0, a_{\dagger}\right)$.

In [8] the main result asserts controllability of the above system to any quasi steady state by a distributed control, except for a small interval of ages near zero. The main open questions raised in the above mentioned reference are the possibility of controlling the whole range of ages and

\footnotetext{
*Institut de Mathématiques de Bordeaux, Université de Bordeaux/Bordeaux INP/CNRS , 351 Cours de la Libération, 33405 TALENCE, France, nicolas.hegoburu@math.u-bordeaux.fr

${ }^{\dagger}$ Institut de Mathématiques de Bordeaux, Université de Bordeaux/Bordeaux INP/CNRS, 351 Cours de la Libération, 33405 TALENCE, France, pierre.magal@math.u-bordeaux.fr

${ }^{\ddagger}$ Institut de Mathématiques de Bordeaux UMR 5251, Université de Bordeaux/Bordeaux INP/CNRS, 351 Cours de la Libération, 33405 TALENCE, France, marius.tucsnak@math.u-bordeaux.fr
} 
designing controls which preserve the positivity of the state trajectories. We give a positive answer to both questions above, in the particular case when the age of individuals able to reproduce is bounded away from zero.

To state our main results, we first recall the standard assumptions, used in particular in [8], on the functions $\mu$ and $\beta$ :

(H1) $\beta \in L^{\infty}\left(0, a_{\dagger}\right), \beta(a) \geqslant 0$ for almost every $a \in\left(0, a_{\dagger}\right)$,

(H2) $\mu \in L^{1}\left[0, a^{*}\right]$ for every $a^{*} \in\left(0, a_{\dagger}\right), \mu(a) \geqslant 0$ for almost every $a \in\left(0, a_{\dagger}\right)$,

(H3) $\int_{0}^{a_{\dagger}} \mu(a) \mathrm{d} a=+\infty$.

The controllability result $[8]$ asserts that if $\delta \in\left(0, a_{0}\right]$ and if $\tilde{p}$ is a time-independant function which satisfies the equations

$$
\left\{\begin{array}{l}
\frac{\partial \tilde{p}}{\partial a}(a)+\mu(a) \tilde{p}(a)=m(a) w(a) \quad\left(a \in\left(0, a_{\dagger}\right)\right), \\
\tilde{p}(0)=\int_{0}^{a_{\dagger}} \beta(a) \tilde{p}(a) \mathrm{d} a
\end{array}\right.
$$

for some function $w \in L^{2}\left[0, a_{\dagger}\right]$, then for every $p_{0} \in L^{2}\left[0, a_{\dagger}\right]$ and every time

$$
a_{\dagger} \leqslant \tau<a_{\dagger}+\delta
$$

there exists $u \in L^{2}\left([0, \tau] ; L^{2}\left[0, a_{\dagger}\right]\right)$ such that

$$
p(\tau, a)=\tilde{p}(a) \quad\left(a \in\left[\delta, a_{\dagger}\right]\right) .
$$

In other words, the above result from [8] asserts that we can control in a time $\tau$ satisfying (1.3) the population of individuals of age at least equal to some $\delta>0$. Our main result asserts that this restriction is not necessary, provided that we assume that the age of individuals able to reproduce is bounded away from zero. More precisely, we have

Theorem 1.1. With the above notation and assumptions, suppose that there exists $a_{b} \in\left(0, a_{\dagger}\right)$ such that

$$
\beta(a)=0 \quad\left(a \in\left(0, a_{b}\right) \text { a.e. }\right) .
$$

Then for every $\tau>a_{\dagger}-a_{0}$, for every $p_{0} \in L^{2}\left(\left[0, a_{\dagger}\right]\right)$ with $p_{0}(a) \geqslant 0$ for almost every $a \in\left(0, a_{\dagger}\right)$, and for any positive function $\tilde{p}$ satisfying $(1.2)$, there exists $u \in L^{2}\left([0, \tau] ; L^{2}\left[0, a_{\dagger}\right]\right)$ such that the solution $p$ of (1.1) satisfies

$$
p(\tau, a)=\tilde{p}(a) \quad\left(a \in\left(0, a_{\dagger}\right) \quad \text { a.e. }\right),
$$

and

$$
p(t, a) \geqslant 0 \text { a.e. }(t, a) \in Q_{\tau} .
$$

For an overview on age structured population dynamics models we refer to Webb [26], Iannelli [13], Kunisch et al [18] and references therein. For related work on size structured population dynamics we refer to Ackleh and Ito [1], Kappel and Ito [14]

The literature devoted to the associated control problems is less abundant but several important results and methods are available. For optimal control problems (namely applied to human population) we refer to Song and $\mathrm{Yu}$ [22]. The null-controllability of the age-dependant population dynamics (1.1) with spatial dependance in the particular case when the control acts for all ages $a$ (the case corresponding to $a_{0}=a_{\dagger}$ ) was investigated by S. Aniţa (see [6], p 148). The case when the control acts in a spatial subdomain $\omega$ and only for small age classes was investigated by B. 
Ainseba and S. Aniţa [3], for initial data $p_{0}$ in a neighborhood of the target $\tilde{p}$. Related approximate and exact controllability issues have also been studied in Ainseba [2], Ainseba and Langlais [5], Ainseba and Iannelli [4], Traore [24], Kavian and Traore [17]. Using a direct approach, the approximate controllability by birth or boundary control is studied in Yu, Guo and Zhu [27].

The remaining part of this work is organized as follows. In Section 2 we recall some basic results on the Lotka-McKendrick semigroup. Section 3 is devoted to the study of the reachable space of (1.1). In Section 4 we investigate some controllability properties of the system (1.1). In particular, we give the proof of Theorem 1.1. In Section 5 we discuss a singular perturbation problem. More precisely we show that if the age interval on which our control is active shrinks to $\{0\}$ the system (including controls and state trajectories) converges to a direct birth controlled system (which can be seen as an "impulse" control). In Section 6 we show how our results apply to a nonlinear system arising in an infection-age model. We end up with an appendix, showing that the considered system is still null controllable in an $L^{1}$ setting.

\section{Some background on the Lotka-McKendrick semigroup}

In this section we recall, with no claim of originality, the formulation of equations (1.1) using semigroup theory.

Denote $X=L^{2}\left[0, a_{\dagger}\right]$ and consider the operator $A: \mathcal{D}(A) \rightarrow X$ defined by

$$
\begin{array}{ll}
\mathcal{D}(A)=\left\{\varphi \in L^{2}\left[0, a_{\dagger}\right]\right. & \left.\varphi(0)=\int_{0}^{a_{\dagger}} \beta(a) \varphi(a) \mathrm{d} a ;-\frac{\mathrm{d} \varphi}{\mathrm{d} a}-\mu \varphi \in L^{2}\left[0, a_{\dagger}\right]\right\}, \\
A \varphi=-\frac{\mathrm{d} \varphi}{\mathrm{d} a}-\mu \varphi & (\varphi \in \mathcal{D}(A)) .
\end{array}
$$

It is well known (see, for instance, Song et al. [23] or Kappel and Zhang [16]) that $A$ generates a $C^{0}$ semigroup of linear operators in $X$ which we denote by $\mathbb{T}=\left(\mathbb{T}_{t}\right)_{t \geqslant 0}$. This allows to define the concept of (mild) solution of (1.1) in the following standard way: we say that $p$ is a mild solution of (1.1) if

$$
p(t, \cdot)=\mathbb{T}_{t} p_{0}+\Phi_{t} u \quad\left(t \geqslant 0, \quad u \in L^{2}([0, \infty) ; X)\right),
$$

where the control operator $B \in \mathcal{L}(X)$ is defined by

$$
B u=m u \quad(u \in X),
$$

and where

$$
\Phi_{t} u=\int_{0}^{t} \mathbb{T}_{t-\sigma} B u(\sigma) \mathrm{d} \sigma \quad\left(t \geqslant 0, \quad u \in L^{2}([0, \infty) ; X)\right)
$$

It is well known (see, for instance, [13] or [26]) that the semigroup $\mathbb{T}$ satisfies

$$
\left(\mathbb{T}_{t} f\right)(a)= \begin{cases}\frac{\pi(a)}{\pi(a-t)} f(a-t) & \text { if } t \leqslant a, \\ \pi(a) b(t-a) & \text { if } t>a,\end{cases}
$$

where $\pi(a)=e^{-\int_{0}^{a} \mu(\sigma) \mathrm{d} \sigma}$ is the probability of survival of an individual from age 0 to $a$ and $b(t)=\left(\mathbb{T}_{t} f\right)(0)=\int_{0}^{a_{\dagger}} \beta(a)\left(\mathbb{T}_{t} f\right)(a) \mathrm{d} a$ is the total birth rate function. Moreover, according, for instance, to $[13, \mathrm{p} .12], b$ is the unique continuous function satisfying the integral Volterra equation of the second kind

$$
b(t)=\int_{0}^{\min \left(t, a_{\dagger}\right)} \beta(t-s) \pi(t-s) b(s) \mathrm{d} s+\int_{\min \left(t, a_{\dagger}\right)}^{a_{\dagger}} \beta(a) \frac{\pi(a)}{\pi(a-t)} f(a-t) \mathrm{d} a .
$$


The state trajectory $p$ of the controlled system (1.1) is given (see, for instance, [8]) by

$$
p(t, a):= \begin{cases}\frac{\pi(a)}{\pi(a-t)} p_{0}(a-t)+c(t, a), & t \leqslant a, \\ \pi(a) \tilde{b}(t-a)+c(t, a), & a<t,\end{cases}
$$

where

$$
c(t, a):= \begin{cases}\int_{a-t}^{a} \frac{\pi(a)}{\pi(s)} m(s) u(s-(a-t), s) \mathrm{d} s, & t \leqslant a, \\ \int_{0}^{a} \frac{\pi(a)}{\pi(s)} m(s) u(s+(t-a), s) \mathrm{d} s, & a<t,\end{cases}
$$

where $\tilde{b}(t)=\int_{0}^{a_{\dagger}} \beta(a) p(t, a) \mathrm{d} a$ is the flux of newly born individuals for the controlled system. It is easy to check that $\tilde{b}$ is the unique continuous function satisfying the Volterra integral equation

$$
\begin{aligned}
\tilde{b}(t) & =\int_{0}^{\min \left(t, a_{\dagger}\right)} \beta(t-s)[\pi(t-s) \tilde{b}(s)+c(t, t-s)] \mathrm{d} s \\
& +\int_{\min \left(t, a_{\dagger}\right)}^{a_{\dagger}} \beta(a)\left[\frac{\pi(a)}{\pi(a-t)} p_{0}(a-t)+c(t, a)\right] \mathrm{d} a .
\end{aligned}
$$

Remark 2.1. The control u constructed in the following section will be of the form

$$
u(t, a)=v(t-a) \quad\left(a \in\left[0, a_{\dagger}\right], t \in[0, \tau]\right),
$$

where $v:\left[-a_{\dagger}, \tau\right] \rightarrow \mathbb{R}$. In this case (2.6) becomes

$$
p(t, a)= \begin{cases}\frac{\pi(a)}{\pi(a-t)} p_{0}(a-t)+v(t-a) \int_{a-t}^{a} \frac{\pi(a)}{\pi(s)} m(s) \mathrm{d} s, & a \geqslant t, \\ \pi(a) \tilde{b}(t-a)+v(t-a) \int_{0}^{a} \frac{\pi(a)}{\pi(s)} m(s) \mathrm{d} s, & a<t .\end{cases}
$$

Remark 2.2. If the state space $X=L^{2}\left[0, a_{\dagger}\right]$ is substituted by $X=L^{1}\left[0, a_{\dagger}\right]$, the operator $A$ : $\mathcal{D}(A) \rightarrow X$ defined by

$$
\begin{array}{ll}
\mathcal{D}(A)=\left\{\varphi \in L^{1}\left[0, a_{\dagger}\right] \quad \mid \quad \varphi(0)=\int_{0}^{a_{\dagger}} \beta(a) \varphi(a) \mathrm{d} a ;-\frac{d \varphi}{d a}-\mu \varphi \in L^{1}\left[0, a_{\dagger}\right]\right\}, \\
A \varphi=-\frac{d \varphi}{d a}-\mu \varphi & (\varphi \in \mathcal{D}(A)),
\end{array}
$$

generates a $C^{0}$ semigroup of linear operators in $X$ denoted by $\mathbb{T}=\left(\mathbb{T}_{t}\right)_{t \geqslant 0}$ (see, for instance, Banks and Kappel [7]). This allows to define the concept of (mild) solution of (1.1) in the same way as above : we say that $p$ is a mild solution of (1.1) if

$$
p(t, \cdot)=\mathbb{T}_{t} p_{0}+\Phi_{t} u \quad\left(t \geqslant 0, \quad u \in L^{1}([0, \infty) ; X)\right),
$$

where the control operator $B \in \mathcal{L}(X)$ is defined by

$$
B u=m u \quad(u \in X),
$$

and where

$$
\Phi_{t} u=\int_{0}^{t} \mathbb{T}_{t-\sigma} B u(\sigma) \mathrm{d} \sigma \quad\left(t \geqslant 0, \quad u \in L^{1}([0, \infty) ; X)\right) .
$$

The semigroup $\mathbb{T}$ satisfies the same formulas (2.4) as in the $L^{2}$ setting. 


\section{Study of the reachable space.}

In this section, we study the reachable space of (1.1) by means of controls $u \in L^{2}\left([0, \tau] ; L^{2}\left[0, a_{\dagger}\right]\right)$. In other words, we study the space Ran $\Phi_{\tau}$, for every $\tau>0$, where the operator $\Phi_{\tau}$ has been defined in (2.3). In order to obtain controllability results, we focus on the case $\tau>a_{\dagger}-a_{0}$ since in the oposite case, as shown below, we have neither approximate nor null controllability. For the sake of completeness, we recall that the property of approximate controllability in some time $\tau>0$ means that Ran $\Phi_{\tau}$ is dense in $X$ and that the null controllability in time $\tau$ means that Ran $\Phi_{\tau} \supset$ Ran $\mathbb{T}_{\tau}$ (we refer, for instance, to [25] - Chapter 11 for a detailed description of these concepts).

Proposition 3.1. Suppose that $\tau<a_{\dagger}-a_{0}$. Then the system (1.1) is neither approximatively controllable, nor null controllable in time $\tau$.

Proof. Let $\tau \in] 0, a_{\dagger}-a_{0}\left[\right.$. Then for every $u \in L^{2}\left([0, \tau] ; L^{2}\left[0, a_{\dagger}\right]\right)$ and for almost every $a \in\left(a_{0}+\right.$ $\left.\tau, a_{\dagger}\right)$, we have from (2.6) that

$$
\left(\Phi_{\tau} u\right)(a)=\int_{a-\tau}^{a} \frac{\pi(a)}{\pi(s)} m(s) u(s+(\tau-a), s) \mathrm{d} s=0,
$$

since $m(s)=0$ if $s \geqslant a-\tau>a_{0}$. This clearly implies that the system (1.1) is not approximately controllable in time $\tau$.

On the other hand, (2.4) implies that there exists $p_{0} \in X$ such that $\left(\mathbb{T}_{\tau} p_{0}\right)(a) \neq 0$ on $\left[a_{0}+\tau, a_{\dagger}\right]$. Consequently, $\operatorname{Ran} \Phi_{\tau} \not \supset \operatorname{Ran} \mathbb{T}_{\tau}$, so that (1.1) is not null controllable in time $\tau$.

The main result in this section is:

Theorem 3.2. Under the assumptions of Theorem 1.1, define

$$
\mathcal{R}:=\left\{\tilde{p} \in X \quad \mid \quad a \mapsto \frac{\tilde{p}(a)}{\pi(a)} \in X, \quad a \mapsto \frac{\frac{\tilde{p}(a)}{\pi(a)}-\int_{0}^{a_{\dagger}-a} \beta(x) \frac{\pi(x)}{\pi(a+x)} \tilde{p}(a+x) \mathrm{d} x}{\int_{0}^{a} \frac{m(x)}{\pi(x)} \mathrm{d} x} \in X\right\} .
$$

Then for every $\tau>a_{\dagger}-a_{0}$ we have $\mathcal{R} \subset \operatorname{Ran} \Phi_{\tau}$.

Proof. We can assume, without loss of generality, that $a_{0} \leqslant a_{b}$ (otherwise we simply control the system for smaller ages). Let $\tau \in\left(a_{\dagger}-a_{0}, a_{\dagger}\right)$.

We define the sets $\left\{P_{\tau}^{j}\right\}_{j} \geqslant-1$ by

$$
P_{\tau}^{-1}=\left\{(t, a) \in(0,+\infty)^{2}: \tau-a_{\dagger} \leqslant t-a \leqslant 0\right\}
$$

and for $j \geqslant 0$

$$
P_{\tau}^{j}=\left\{(t, a) \in(0,+\infty)^{2}: j a_{b}<t-a \leqslant(j+1) a_{b}\right\} .
$$

Given $\tilde{p} \in \mathcal{R}$, we look for $v \in L^{2}\left[-a_{\dagger}, \tau\right]$ such that setting $u(t, a)=v(t-a)$ for every $t \in[0, \tau]$ and $a \in\left[0, a_{\dagger}\right]$ we have

$$
\Phi_{\tau} u(a)=\tilde{p}(a) \quad\left(a \in\left[0, a_{\dagger}\right] \quad \text { a.e. }\right) .
$$

Case $t-a<\tau-a_{\dagger}:$ We first set $v(s)=0$ for $s \in\left[-a_{\dagger}, \tau-a_{\dagger}\right]$ so that, according to (2.9) we have

$$
\left(\Phi_{t} u\right)(a)=0 \quad\left(t-a<\tau-a_{\dagger}\right) .
$$




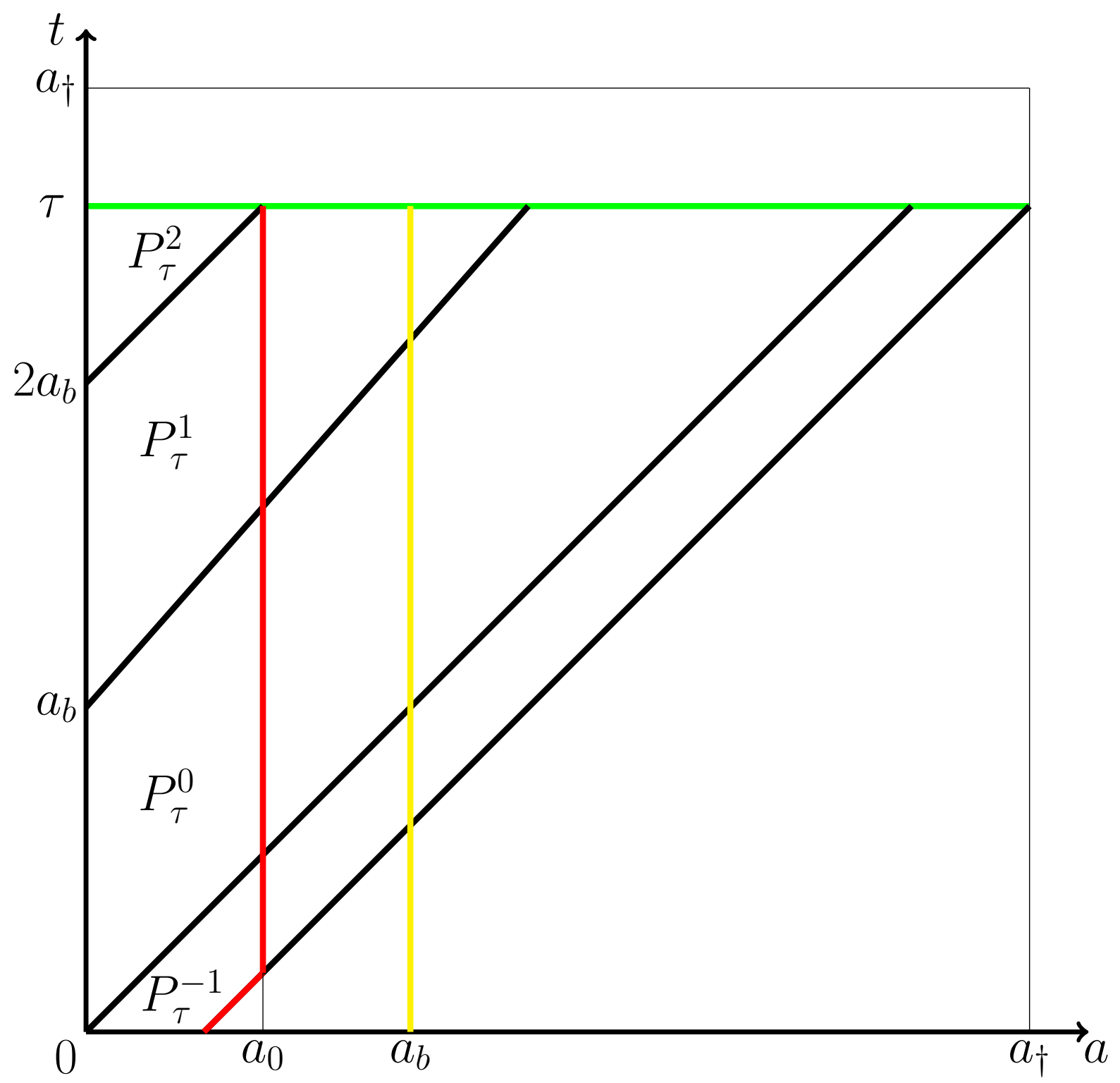

Figure 1: In this figure the control possibly acts in the subset of $Q_{\tau}=[0, \tau] \times\left[0, a_{\dagger}\right]$ delimited by the line $a=0$ and the red curve. The domain $Q_{\tau}$ is decomposed into subregions $P_{\tau}^{-1}, P_{\tau}^{0}, P_{\tau}^{1}, P_{\tau}^{2}, \ldots$ which are delimited respectively by the characteristics lines $t-a=\tau-a_{\dagger}, t-a=0, t-a=a_{b}$, $t-a=2 a_{b}, \ldots$ For each subregion $P_{\tau}^{j}$ (for $j \geqslant 0$ ) the values of $\tilde{b}(t)=\int_{0}^{a_{\dagger}} \beta(a) p(t, a) \mathrm{d} a$ for $t \in\left[j a_{b},(j+1) a_{b}\right]$ depends only on the control in the previous subregions $P_{\tau}^{-1}, \ldots, P_{\tau}^{j-1}$. This will allow us, using an inductive method, to derive an explicit formula of the control functions in terms of the targeted distribution of population.

Case $\tau-a_{\dagger} \leqslant t-a \leqslant 0$ : By using again (2.9), it follows that

$$
\left(\Phi_{\tau} u\right)(a)=v(\tau-a) \int_{a-\tau}^{a} \frac{\pi(a)}{\pi(s)} m(s) \mathrm{d} s \quad\left(a \in\left[\tau, a_{\dagger}\right] \quad \text { a.e. }\right)
$$

Consequently, (3.3) is satisfied for $a \in\left[\tau, a_{\dagger}\right]$ iff

$$
v(s)=\frac{\frac{\tilde{p}(\tau-s)}{\pi(\tau-s)}}{\int_{-s}^{\tau-s} \frac{m(x)}{\pi(x)} \mathrm{d} x} \quad\left(s \in\left[\tau-a_{\dagger}, 0\right] \quad \text { a.e. }\right) .
$$


Note that, since $\tau>a_{\dagger}-a_{0}, s \in\left[\tau-a_{\dagger}, 0\right]$ implies that $-s<a_{0}$ therefore for every $s \in\left[\tau-a_{\dagger}, 0\right]$ we have

$$
\int_{-s}^{\tau-s} \frac{m(x)}{\pi(x)} \mathrm{d} x>0
$$

Moreover by assumption we have $a \mapsto \frac{\tilde{p}(a)}{\pi(a)} \in L^{2}\left[0, a_{\dagger}\right]$ hence

$$
v \in L^{2}\left[\tau-a_{\dagger}, 0\right] .
$$

Case $0<t-a<a_{b}$ : According to (2.9) and (3.5), for almost every $(t, a) \in P_{\tau}^{-1}$ we have

$$
\begin{aligned}
\left(\Phi_{t} u\right)(a) & =v(t-a) \int_{a-t}^{a} \frac{\pi(a)}{\pi(s)} m(s) \mathrm{d} s \\
& =\pi(a) \frac{\int_{a-t}^{a} \frac{m(s)}{\pi(s)} \mathrm{d} s}{\int_{a-t}^{\tau+(a-t)} \frac{m(s)}{\pi(s)} \mathrm{d} s} \frac{\tilde{p}(\tau+(a-t))}{\pi(\tau+(a-t))} .
\end{aligned}
$$

To determine $v$ on $\left[0, a_{b}\right]$ we note that from (2.9) it follows that

$$
\left(\Phi_{\tau} u\right)(a)=\pi(a) \tilde{b}(\tau-a)+v(\tau-a) \int_{0}^{a} \frac{\pi(a)}{\pi(s)} m(s) \mathrm{d} s \quad\left(a \in\left[\tau-a_{b}, \tau\right] \quad \text { a.e. }\right),
$$

where

$$
\tilde{b}(t)=\int_{a_{b}}^{a_{\dagger}} \beta(a)\left(\Phi_{t} u\right)(a) \mathrm{d} a \quad\left(t \in\left[0, a_{b}\right]\right) .
$$

To determine $\tilde{b}$, after some simple calculations and using (3.4), (3.6) and (3.8) it follows that

$$
\tilde{b}(t)=\int_{a_{b}}^{t+a_{\dagger}-\tau} \beta(a) \pi(a) \frac{\tilde{p}(\tau+(a-t))}{\pi(\tau+(a-t))} \mathrm{d} a \quad\left(t \in\left[0, a_{b}\right]\right) .
$$

Inserting the above formula (3.9) in (3.7) it follows that

$$
\left(\Phi_{\tau} u\right)(a)=\pi(a) \int_{a_{b}}^{a_{\dagger}-a} \beta(x) \pi(x) \frac{\tilde{p}(a+x)}{\pi(a+x)} \mathrm{d} x+v(\tau-a) \int_{0}^{a} \frac{\pi(a)}{\pi(s)} m(s) \mathrm{d} s \quad\left(a \in\left[\tau-a_{b}, \tau\right] \text { a.e. }\right) .
$$

Thus, (3.3) is satisfied for $a \in\left[\tau-a_{b}, \tau\right]$ iff

$$
v(s)=\frac{\tilde{p}(\tau-s)-\pi(\tau-s) \chi(s)}{\pi(\tau-s) \int_{0}^{\tau-s} \frac{m(x)}{\pi(x)} \mathrm{d} x} \quad\left(s \in\left[0, a_{b}\right] \quad \text { a.e. }\right),
$$

where

$$
\chi(s):=\int_{a_{b}}^{s+a_{\dagger}-\tau} \beta(x) \pi(x) \frac{\tilde{p}(\tau-(s-x))}{\pi(\tau-(s-x))} \mathrm{d} x \quad(s \in[0, \tau]) .
$$

Since $\tilde{p} \in L^{2}\left[0, a_{\dagger}\right]$, we clearly have that $v \in L^{2}\left[0, a_{b}\right]$.

Case $j a_{b}<t-a<(j-1) a_{b}$ for some integer $j \geqslant 1$ : The above calculations suggest as possible control driving the zero initial state to $u(t, a)=v(t-a)$ where

$$
v(s)=\left\{\begin{array}{lll}
0 & \text { for } & s \in\left[-a_{\dagger}, \tau-a_{\dagger}\right], \\
\frac{\frac{\tilde{p}(\tau-s)}{\pi(\tau-s)}}{\int_{-\frac{s}{\tau(x)} \frac{m(x)}{\pi(x)} \mathrm{d} x}^{\tilde{p}(\tau-s)-\pi(\tau-s) \chi(s)}} & \text { for } & s \in\left[\tau-a_{\dagger}, 0\right], \\
\frac{\pi(\tau-s) \int_{0}^{\tau-s} \frac{m(x)}{\pi(x)} \mathrm{d} x}{\pi(\tau-s)} & \text { for } & s \in[0, \tau] .
\end{array}\right.
$$


where $\chi(s)$ is defined by $(3.12)$.

To prove this fact we first note that $v$ defined above is in $L^{2}\left[-a_{\dagger}, \tau\right]$ due to the fact that $\tilde{p} \in \mathcal{R}$. Moreover, we have already seen that (3.13) gives

$$
\left(\Phi_{\tau} u\right)(a)=\tilde{p}(a) \quad\left(a \in\left[\tau-a_{b}, a_{\dagger}\right] \quad \text { a.e. }\right) .
$$

We next show by induction over $j \geqslant 0$ the following properties which we denote by $\left(\mathcal{P}_{j}\right)$ :

- $\tilde{b}(t)=\chi(t)$ for every $t \in\left[j a_{b},(j+1) a_{b}\right]$,

- $\left(\Phi_{\tau} u\right)(a)=\tilde{p}(a)$ for a.e. $a \in\left(\tau-(j+1) a_{b}, \tau-j a_{b}\right)$.

We have seen above that $\left(\mathcal{P}_{0}\right)$ is true.

Let $j \geqslant 0$ be an integer fixed, and suppose that $\left(\mathcal{P}_{i}\right)$ holds for every $i \in\{0, \ldots, j\}$. Then, for every $i \in\{0, \ldots, j\}$ and for almost every $(t, a) \in P_{\tau}^{i}$, from (2.9) and (3.13) we have

$$
\left(\Phi_{t} u\right)(a)=\pi(a) \chi(t-a)\left[1-\frac{\int_{0}^{a} \frac{m(s)}{\pi(s)} \mathrm{d} s}{\int_{0}^{\tau-(t-a)} \frac{m(s)}{\pi(s)} \mathrm{d} s}\right]+\pi(a) \frac{\int_{0}^{a} \frac{m(x)}{\pi(x)} \mathrm{d} x}{\int_{0}^{\tau-(t-a)} \frac{m(x)}{\pi(x)} \mathrm{d} x} \frac{\tilde{p}(\tau-(t-a))}{\pi(\tau-(t-a))} .
$$

Combining (3.4), (3.6) and the above formula (3.14), we are in position to compute $\tilde{b}(t)$ for $t \in$ $\left[(j+1) a_{b},(j+2) a_{b}\right]$. Indeed, since $\tilde{b}(t)=\int_{a_{b}}^{a_{\dagger}} \beta(a)\left(\Phi_{t} u\right)(a) \mathrm{d} a$ for every $t \in\left[(j+1) a_{b},(j+2) a_{b}\right]$, we can use (3.4) to obtain that

$$
\tilde{b}(t)=\int_{a_{b}}^{t} \beta(a)\left(\Phi_{t} u\right)(a) \mathrm{d} a+\int_{t}^{t+a_{\dagger}-\tau} \beta(a)\left(\Phi_{t} u\right)(a) \mathrm{d} a \quad\left(t \in\left[(j+1) a_{b},(j+2) a_{b}\right]\right) .
$$

Firstly, according to (3.14), the first integral in the above formula (3.15) writes

$$
\begin{aligned}
\int_{a_{b}}^{t} \beta(a)\left(\Phi_{t} u\right)(a) \mathrm{d} a=\int_{a_{b}}^{t} \beta(a)(\pi(a) \chi(t-a) & {\left[1-\frac{\int_{0}^{a} \frac{m(s)}{\pi(s)} \mathrm{d} s}{\left.\int_{0}^{\tau-(t-a)} \frac{m(s)}{\pi(s)} \mathrm{d} s\right]}\right.} \\
+\pi(a) & {\left.\left[\frac{\int_{0}^{a} \frac{m(x)}{\pi(x)} \mathrm{d} x}{\int_{0}^{\tau-(t-a)} \frac{m(x)}{\pi(x)} \mathrm{d} x}\right] \frac{\tilde{p}(\tau-(t-a))}{\pi(\tau-(t-a))}\right) \mathrm{d} a . }
\end{aligned}
$$

We note that the term contained in the first bracket of the above formula involves only values of $a$ which are larger than $a_{0}$, so it vanishes. Moreover the term in the second bracket of the last formula is clearly equal to one. Consequently,

$$
\int_{a_{b}}^{t} \beta(a)\left(\Phi_{t} u\right)(a) \mathrm{d} a=\int_{a_{b}}^{t} \beta(a) \pi(a) \frac{\tilde{p}(\tau+(a-t))}{\pi(\tau+(a-t))} \mathrm{d} a \quad\left(t \in\left[(j+1) a_{b},(j+2) a_{b}\right]\right) .
$$

Moreover, by using (3.6) it is not difficult to check that for every $t \in\left[(j+1) a_{b},(j+2) a_{b}\right]$,

$$
\int_{t}^{t+a_{\dagger}-\tau} \beta(a)\left(\Phi_{t} u\right)(a) \mathrm{d} a=\int_{t}^{t+a_{\dagger}-\tau} \beta(a) \pi(a) \frac{\tilde{p}(\tau+(a-t))}{\pi(\tau+(a-t))} \mathrm{d} a .
$$


Using (3.15), (3.16) and (3.17) we have

$$
\tilde{b}(t)=\int_{a_{b}}^{t+a_{\dagger}-\tau} \beta(a) \pi(a) \frac{\tilde{p}(\tau+(a-t))}{\pi(\tau+(a-t))} \mathrm{d} a=\chi(t) \quad\left(t \in\left[(j+1) a_{b},(j+2) a_{b}\right]\right) .
$$

Consequently, if $(j+1) a_{b}<t-a \leq(j+2) a_{b}$, we obtain from (2.9), (3.13) and (3.18) that

$$
\left(\Phi_{t} u\right)(a)=\pi(a) \chi(t-a)\left[1-\frac{\int_{0}^{a} \frac{m(s)}{\pi(s)} \mathrm{d} s}{\int_{0}^{\tau-(t-a)} \frac{m(s)}{\pi(s)} \mathrm{d} s}\right]+\pi(a) \frac{\int_{0}^{a} \frac{m(x)}{\pi(x)} \mathrm{d} x}{\int_{0}^{\tau-(t-a)} \frac{m(x)}{\pi(x)} \mathrm{d} x} \frac{\tilde{p}(\tau-(t-a))}{\pi(\tau-(t-a))} .
$$

The above formula implies that $\left(\Phi_{\tau} u\right)(a)=\tilde{p}(a)$ for almost every $a \in\left(\tau-(j+2) a_{b}, \tau-(j+1) a_{b}\right)$, which ends the induction proof.

Remark 3.3. Given $\tau \in] a_{\dagger}-a_{0}, a_{\dagger}[$ and $\tilde{p} \in \mathcal{R}$, the proof of Theorem 3.2 gives an explicit formula for a control $u \in L^{2}\left([0, \tau] ; L^{2}\left[0, a_{\dagger}\right]\right)$ satisfying $\Phi_{\tau} u=\tilde{p}$. More precisely, for almost every $(t, a) \in Q_{\tau}$ we have

$$
u(t, a)=\left\{\begin{array}{lr}
0 & \text { if } t-a<\tau-a_{\dagger}, \\
\frac{\frac{\tilde{p}(\tau+(a-t))}{\pi(\tau+(a-t))}}{\int_{a-t}^{\tau+(a-t)} \frac{m(x)}{\pi(x)} \mathrm{d} x} & \text { if } \quad \tau-a_{\dagger} \leqslant t-a \leqslant 0, \\
\frac{\tilde{p}(\tau-(t-a))-\pi(\tau-(t-a)) \chi(t-a)}{\pi(\tau-(t-a)) \int_{0}^{\tau-(t-a)} \frac{m(x)}{\pi(x)} \mathrm{d} x} & \text { if } 0<t-a \leqslant \tau,
\end{array}\right.
$$

where

$$
\chi(s)=\int_{a_{b}}^{s+a_{\dagger}-\tau} \beta(x) \pi(x) \frac{\tilde{p}(\tau-(s-x))}{\pi(\tau-(s-x))} \mathrm{d} x \quad(s \in[0, \tau]) .
$$

Moreover, for almost every $(t, a) \in Q_{\tau}$, we have

$$
\left(\Phi_{t} u\right)(a)= \begin{cases}0 & \text { if } t-a<\tau-a_{\dagger}, \\ \pi(a) \frac{\int_{a-t}^{a} \frac{m(s)}{\pi(s)} \mathrm{d} s}{\int_{a-t}^{\tau+(a-t)} \frac{m(s)}{\pi(s)} \mathrm{d} s} \frac{\tilde{p}(\tau+(a-t))}{\pi(\tau+(a-t))} & \text { if } \quad \tau-a_{\dagger} \leqslant t-a \leqslant 0, \\ \pi(a) \chi(t-a)\left[1-\frac{\int_{0}^{a} \frac{m(s)}{\pi(s)} \mathrm{d} s}{\left.\int_{0}^{\tau-(t-a)} \frac{m(s)}{\pi(s)} \mathrm{d} s\right]}\right. & \\ +\pi(a) \frac{\int_{0}^{a} \frac{m(x)}{\pi(x)} \mathrm{d} x}{\int_{0}^{\tau-(t-a)} \frac{m(x)}{\pi(x)} \mathrm{d} x} \frac{\tilde{p}(\tau-(t-a))}{\pi(\tau-(t-a))} & \text { if } 0<t-a \leqslant \tau .\end{cases}
$$

Remark 3.4. For every $\varphi \in \mathcal{D}(A)$ with $\frac{\varphi}{\pi} \in X$, we have $\varphi \in \mathcal{R}$. Indeed, for every $\varphi \in \mathcal{D}(A)$, there exists $f \in X$ such that $\varphi^{\prime}+\mu \varphi=f$, so that assuming the condition $\frac{\varphi}{\pi} \in X$, for almost every 
$a \in\left(0, a_{\dagger}\right)$ we have

$$
\begin{aligned}
\frac{\varphi(a)}{\pi(a)} & =\varphi(0)+\int_{0}^{a} \frac{\varphi^{\prime}(s) \pi(s)+\mu(s) \pi(s) \varphi(s)}{\pi^{2}(s)} \mathrm{d} s \\
& =\int_{0}^{a_{\dagger}} \beta(a) \varphi(a) \mathrm{d} a+\int_{0}^{a} \frac{f(s)}{\pi(s)} \mathrm{d} s .
\end{aligned}
$$

Denoting by $I:=\int_{0}^{a_{\dagger}} \beta(a) \varphi(a) \mathrm{d} a$ and $i(a)=\int_{0}^{a_{\dagger}-a} \beta(x) \frac{\pi(x)}{\pi(a+x)} \varphi(a+x) \mathrm{d} x$, it follows from the above formula (3.19) that we have

$$
\frac{\varphi(a)}{\pi(a)}-\int_{0}^{a_{\dagger}-a} \beta(x) \frac{\pi(x)}{\pi(a+x)} \varphi(a+x) \mathrm{d} x=\int_{0}^{a} \frac{f(s)}{\pi(s)} \mathrm{d} s+I-i(a) .
$$

Let us show that the map

$$
a \mapsto \frac{\int_{0}^{a} \frac{f(s)}{\pi(s)} \mathrm{d} s+I-i(a)}{a}
$$

is in $L^{2}\left[0, a_{\dagger}\right]$. By Hardy's inequality (see [12, $p$ 240]), the map $a \mapsto \frac{\int_{0}^{a} \frac{f(s)}{\pi(s)} \mathrm{d} s}{a}$ lies in $L^{2}\left[0, a_{\dagger}\right]$. For the second term at the numerator in (3.20) we note that for almost every $a \in\left(0, a_{\dagger}\right)$ we have

$$
\frac{\mathrm{d}}{\mathrm{d} a}(I-i(a))=-\beta\left(a_{\dagger}-a\right) \pi\left(a_{\dagger}-a\right)\left(\varphi(0)+\int_{0}^{a_{\dagger}} \frac{f(s)}{\pi(s)} \mathrm{d} s\right)-\int_{0}^{a_{\dagger}-a} \beta(x) \pi(x) \frac{f(a+x)}{\pi(a+x)} \mathrm{d} x,
$$

so that the map $a \mapsto \frac{\mathrm{d}}{\mathrm{d} a}(I-i(a))$ is in $L^{2}\left[0, a_{\dagger}\right]$. It follows that the map $F: a \mapsto I-i(a)$ satisfies $F \in H^{1}\left[0, a_{\dagger}\right]$ with $F(0)=0$, so that from Hardy's inequality (see [12, $p$ 240]) the map $a \mapsto \frac{F(a)}{a}$ is in $L^{2}\left[0, a_{\dagger}\right]$. Consequently, from (3.20) we have $\varphi \in \mathcal{R}$.

\section{Some controllability results}

In this section we investigate some controllability properties of the system (1.1).

We first note that the following null controllability result holds.

Proposition 4.1. For every $\tau>a_{\dagger}-a_{0}$, we have $\operatorname{Ran} \mathbb{T}_{\tau} \subset \mathcal{R}$, where $\mathcal{R}$ has been defined in (3.2). Consequently, if $\beta$ satisfies (1.4) then the system (1.1) is null controllable in any time $\tau>a_{\dagger}-a_{0}$.

Proof. Without loss of generality, we can assume that $a_{\dagger}-a_{0}<\tau<a_{\dagger}$. Indeed, for $\tau \geqslant a_{\dagger}$, the result follows using the semigroup property and replacing $p_{0}$ by $\mathbb{T}_{\varepsilon} p_{0}$ for some convenient $\varepsilon>0$. Let $\tilde{p} \in \operatorname{Ran} \mathbb{T}_{\tau}$. Then from (2.4) there exists $p_{0} \in X$ such that

$$
\tilde{p}(a)=\left\{\begin{array}{ll}
\frac{\pi(a)}{\pi(a-\tau)} p_{0}(a-\tau) & \text { if } \tau \leqslant a, \\
\pi(a) b(\tau-a) & \text { if } \tau>a,
\end{array} \quad\left(a \in\left[0, a_{\dagger}\right] \quad \text { a.e. }\right),\right.
$$

with $b(t)=\int_{0}^{a_{\dagger}} \beta(a)\left(\mathbb{T}_{t} p_{0}\right)(a) \mathrm{d} a$. Consequently, for almost every $a \in(0, \tau)$ we have $\frac{\tilde{p}(a)}{\pi(a)}=$ $b(\tau-a)$ and for almost every $a \in\left(\tau, a_{\dagger}\right)$ we have $\frac{\tilde{p}(a)}{\pi(a)}=\frac{p_{0}(a-\tau)}{\pi(a-\tau)}$. Hence, the function $a \mapsto \frac{\tilde{p}}{\pi}$ is in $L^{2}\left[0, a_{\dagger}\right]$. From that it is easy to check that

$$
a \mapsto \frac{\frac{\tilde{p}(a)}{\pi(a)}-\int_{0}^{a_{\dagger}-a} \beta(x) \frac{\pi(x)}{\pi(a+x)} \tilde{p}(a+x) \mathrm{d} x}{\int_{0}^{a} \frac{m(x)}{\pi(x)} \mathrm{d} x},
$$


is in $L^{2}\left[\varepsilon, a_{\dagger}\right]$ for every $\varepsilon>0$. To show that $\tilde{p} \in \mathcal{R}$, we still have to check that the map defined in (4.2) is in $L^{2}[0, \varepsilon]$ for every $\varepsilon>0$ small enough. To this aim, applying first (2.4) we note that, for almost every $a \in(0, \varepsilon)$,

$$
\begin{aligned}
\frac{\tilde{p}(a)}{\pi(a)}- & \int_{0}^{a_{\dagger}-a} \beta(x) \frac{\pi(x)}{\pi(a+x)} \tilde{p}(a+x) \mathrm{d} x \\
& =b(\tau-a)-\int_{0}^{a_{\dagger}-a} \beta(x) \frac{\pi(x)}{\pi(a+x)}\left(\mathbb{T}_{\tau} p_{0}\right)(a+x) \mathrm{d} x \\
& =b(\tau-a)-\int_{0}^{\tau-a} \beta(x) \frac{\pi(x)}{\pi(a+x)}\left(\mathbb{T}_{\tau} p_{0}\right)(a+x) \mathrm{d} x-\int_{\tau-a}^{a_{\dagger}-a} \beta(x) \frac{\pi(x)}{\pi(a+x)}\left(\mathbb{T}_{\tau} p_{0}\right)(a+x) \mathrm{d} x \\
& =b(\tau-a)-\int_{0}^{\tau-a} \beta(x) \pi(x) b(\tau-(a+x)) \mathrm{d} x-\int_{\tau-a}^{a_{\dagger}-a} \beta(x) \pi(x) \frac{p_{0}(a+x-\tau)}{\pi(a+x-\tau)} \mathrm{d} x .
\end{aligned}
$$

Moreover, using (2.5) it follows that for every $a \in(0, \varepsilon)$ we have

$$
b(\tau-a)=\int_{0}^{\tau-a} \beta(x) \pi(x) b(\tau-(a+x)) \mathrm{d} x+\int_{\tau-a}^{a_{\dagger}} \beta(x) \pi(x) \frac{p_{0}(a+x-\tau)}{\pi(a+x-\tau)} \mathrm{d} x .
$$

We can thus combine the last two formulas to obtain that for almost every $a \in(0, \varepsilon)$ we have

$$
\frac{\tilde{p}(a)}{\pi(a)}-\int_{0}^{a_{\dagger}-a} \beta(x) \frac{\pi(x)}{\pi(a+x)} \tilde{p}(a+x) \mathrm{d} x=\int_{a_{\dagger}-a}^{a_{\dagger}} \beta(x) \pi(x) \frac{p_{0}(a+x-\tau)}{\pi(a+x-\tau)} \mathrm{d} x .
$$

Since $\beta \in L^{\infty}\left[0, a_{\dagger}\right]$ and $\pi$ is a decreasing function, it follows that for almost every $a \in(0, \varepsilon)$,

$$
\left|\frac{\tilde{p}(a)}{\pi(a)}-\int_{0}^{a_{\dagger}-a} \beta(x) \frac{\pi(x)}{\pi(a+x)} \tilde{p}(a+x) \mathrm{d} x\right| \leqslant\|\beta\|_{L^{\infty}\left[0, a_{\dagger}\right]} \int_{a_{\dagger}-\tau}^{a_{\dagger}-\tau+a}\left|p_{0}(s)\right| \mathrm{d} s .
$$

On the other hand, Hardy's inequality (see, for instance, [12, p. 240]) ensures that

$$
a \mapsto \frac{\int_{0}^{a}\left|p_{0}\left(s+\left(a_{\dagger}-\tau\right)\right)\right| \mathrm{d} s}{a} \in L^{2}[0, \varepsilon],
$$

hence $\tilde{p} \in \mathcal{R}$.

To obtain the null controllability assertion it suffices to combine the inclusions Ran $\mathbb{T}_{\tau} \subset \mathcal{R}$ which we have just proved and $\mathcal{R} \subset \operatorname{Ran} \Phi_{\tau}$ which was proved in Theorem 3.2.

The result below and the following one answer to an open question raised in [8] and it essentially asserts that, with an extra assumption, the system can be "positively" steered from any positive initial state in $X$ to any positive state in $\mathcal{R}$ by means of a $L^{2}$ control. More precisely, we have,

Theorem 4.2. Let the assumptions of Theorem 1.1 be satisfied. Let $p_{0} \in X$ and $\tilde{p} \in \mathcal{R}$. Then for every $\tau>a_{\dagger}-a_{0}$, there exists a control $u \in L^{2}\left([0, \tau] ; L^{2}\left[0, a_{\dagger}\right]\right)$ such that the controlled solution $p$ of (1.1) satisfies

$$
p(\tau, a)=\tilde{p}(a) \quad\left(a \in\left(0, a_{\dagger}\right) \quad \text { a.e. }\right) .
$$

Moreover, if $p_{0}(a) \geqslant 0$ and $\tilde{p}(a) \geqslant 0$ for almost every $a \in\left(0, a_{\dagger}\right)$, then the control can be chosen such that the controlled state trajectory remains positive, i.e., such that for every $t \in[0, \tau]$ we have

$$
p(t, a) \geqslant 0 \quad\left(a \in\left[0, a_{\dagger}\right] \text { a.e. }\right) .
$$

Proof. We give the proof in the case $\tau \in\left(a_{\dagger}-a_{0}, a_{\dagger}\right)$. 
We set $z=p-\tilde{p}$. The aim is to find a control function $u \in L^{2}\left([0, \tau] ; L^{2}\left[0, a_{\dagger}\right]\right)$ such as $z(\tau)=0$ and $p(t, a) \geqslant 0$ for almost every $(t, a) \in Q_{\tau}$. To achieve this goal we set

$$
z(t)=\mathbb{T}_{t} p_{0}+\Phi_{t} u-\tilde{p} \quad(t \geqslant 0) .
$$

Since from Theorem 3.2 and Proposition 4.1 we have $\tilde{p}-\mathbb{T}_{\tau} p_{0} \in \mathcal{R}$ we can aply the construction in Theorem 3.2 to obtain a control $u \in L^{2}\left([0, \tau] ; L^{2}\left[0, a_{\dagger}\right]\right)$ such as

$$
\Phi_{\tau} u=\tilde{p}-\mathbb{T}_{\tau} p_{0} .
$$

The corresponding state trajectory will satisfy, in particular, the condition $p(\tau, \cdot)=\tilde{p}$.

To prove the positivity of the state trajectory constructed above we first assume that $p_{0}$ and $\tilde{p}$ are continuous on $\left[0, a_{\dagger}\right]$. Then the the control $u$ described in Remark 3.3 is of the form $u(t, a)=v(t-a)$, with $v$ continuous on each one of the intervals $\left[-a_{\dagger}, \tau-a_{\dagger}\right],\left[\tau-a_{\dagger}, 0\right]$ and $[0, \tau]$. Moreover, the corresponding state trajectory $p$, also described in Remark 3.3, is continuous on each one of the domains $Q_{\tau}^{1}, Q_{\tau}^{2}$ and $Q_{\tau}^{3}$, where

$$
\begin{gathered}
Q_{\tau}^{1}=\left\{(t, a) \in Q_{\tau} \mid-a_{\dagger} \leqslant t-a<\tau-a_{\dagger}\right\}, \\
Q_{\tau}^{2}=\left\{(t, a) \in Q_{\tau} \mid \tau-a_{\dagger} \leqslant t-a \leqslant 0\right\}, \\
Q_{\tau}^{3}=\left\{(t, a) \in Q_{\tau} \mid 0<t-a \leqslant \tau\right\} .
\end{gathered}
$$

Since the initial condition $p_{0}$ is supposed positive and the control $u$ vanishes on $Q_{\tau}^{1}$, the solution $p$ of the controlled system (1.1) is positive on $Q_{\tau}^{1}$. Indeed, from (2.9) we have

$$
p(t, a)=\frac{\pi(a)}{\pi(a-t)} p_{0}(a-t) \quad\left((t, a) \in Q_{\tau}^{1}\right) .
$$

Moreover, if $(t, a) \in Q_{\tau}^{2}$ with $a \geqslant a_{0}$, using (2.9) we have

$$
p(t, a)=\frac{\pi(a)}{\pi(a-t)} p_{0}(a-t)+v(t-a) \int_{a-t}^{a_{0}} \frac{\pi(a)}{\pi(s)} \mathrm{d} s \quad\left((t, a) \in Q_{\tau}^{2}, a \geqslant a_{0}\right) .
$$

It follows from the above formula that for every $(t, a) \in Q_{\tau}^{2}$ with $a \geqslant a_{0}$ we have

$$
\begin{aligned}
\tilde{p}(\tau-(t-a))=p(\tau, \tau- & (t-a))=\frac{\pi(\tau-(t-a))}{\pi(a-t)} p_{0}(a-t) \\
& +v(t-a) \int_{a-t}^{a_{0}} \frac{\pi(\tau-(t-a))}{\pi(s)} \mathrm{d} s \quad\left((t, a) \in Q_{\tau}^{2}, a \geqslant a_{0}\right) .
\end{aligned}
$$

By combining (4.5) and (4.6) we get

$$
p(t, a)=\frac{\pi(a)}{\pi(\tau-(t-a))} \tilde{p}(\tau-(t-a)) \quad\left((t, a) \in Q_{\tau}^{2}, a \geqslant a_{0}\right) .
$$

Since the target $\tilde{p}$ is supposed positive, it follows from (4.7) that the solution $p$ of the controlled system (1.1) is positive on the subdomain $\left\{(t, a) \in Q_{\tau}^{2}, a \geqslant a_{0}\right\}$.

If $(t, a) \in Q_{\tau}^{3}$ with $a \geqslant a_{0}$, using (2.9) we have

$$
p(t, a)=\pi(a) \tilde{b}(t-a)+v(t-a) \int_{0}^{a_{0}} \frac{\pi(a)}{\pi(s)} \mathrm{d} s \quad\left((t, a) \in Q_{\tau}^{3}, a \geqslant a_{0}\right) .
$$

It follows from the above formula that for every $(t, a) \in Q_{\tau}^{3}$ with $a \geqslant a_{0}$ we have

$$
\tilde{p}(\tau-(t-a))=\pi(\tau-(t-a)) \tilde{b}(t-a)+v(t-a) \int_{0}^{a_{0}} \frac{\pi(\tau-(t-a))}{\pi(s)} \mathrm{d} s \quad\left((t, a) \in Q_{\tau}^{3}, a \geqslant a_{0}\right),
$$


so that using (4.8) and (4.9) we get

$$
p(t, a)=\frac{\pi(a)}{\pi(\tau-(t-a))} \tilde{p}(\tau-(t-a)) \quad\left((t, a) \in Q_{\tau}^{3}, a \geqslant a_{0}\right) .
$$

Since the target $\tilde{p}$ is supposed positive, it follows from (4.10) that the solution $p$ of the controlled system (1.1) is positive on the subdomain $\left\{(t, a) \in Q_{\tau}^{3}, a \geqslant a_{0}\right\}$. In particular, using (4.7) and (4.10), the solution $p$ is positive along the red curve represented in Figure 1.

It remains to show the positivity of the controlled solution $p$ into the subregions $\left\{(t, a) \in Q_{\tau}^{2}, a \leqslant\right.$ $\left.a_{0}\right\}$ and $\left\{(t, a) \in Q_{\tau}^{3}, a \leqslant a_{0}\right\}$. To this aim, note first that if $(t, a) \in Q_{\tau}^{2}$ with $a \leqslant a_{0}$, then from (2.9) we have

$$
p(t, a)=\pi(a)\left(\frac{p_{0}(a-t)}{\pi(a-t)}+v(t-a) \int_{a-t}^{a} \frac{m(s)}{\pi(s)} \mathrm{d} s\right) \quad\left((t, a) \in Q_{\tau}^{2}, a \leqslant a_{0}\right) .
$$

Let us show that the density $p(t, a)$ is positive along each characteristic line $t-a=c$, with $c \in\left[\tau-a_{\dagger}, 0\right]$ and $a \leqslant a_{0}$. Note first that if $c \in\left[\tau-a_{\dagger}, 0\right]$, then for every $(t, a) \in Q_{\tau}^{2}$ with $a \leqslant a_{0}$ and $t-a=c$, from (4.11) we have

$$
p(a+c, a)=\pi(a)\left(\frac{p_{0}(-c)}{\pi(-c)}+v(c) \int_{-c}^{a} \frac{m(s)}{\pi(s)} \mathrm{d} s\right) .
$$

If $v(c) \geqslant 0$, then from the above formula (4.12), the density $p(t, a)$ is clearly positive along the characteristic line $t-a=c$. If $v(c)<0$, the control acts like a retrieval of individuals so that along the characteristic line $t-a=c$, the population density decreases and must achieve its minimum value on the red vertical line represented in Figure 1, minimum which is positive due to (4.7) and the fact that the solution $p$ is continuous on $Q_{\tau}^{2}$. In fact, with $c$ fixed in $\left[\tau-a_{\dagger}, 0\right]$, in the case where $v(c)<0$, the quantity

$$
\frac{p_{0}(-c)}{\pi(-c)}+v(c) \int_{-c}^{a} \frac{m(s)}{\pi(s)} \mathrm{d} s
$$

is a decreasing expression of $a$ which attains its positive minimum for $a=a_{0}$. It follows that $p(t, a) \geqslant 0$ for almost every $(t, a) \in Q_{\tau}^{2}$ with $a \leqslant a_{0}$.

Similar arguments can be used to show the positivity of the solution $p$ on $\left\{(t, a) \in Q_{\tau}^{3}, a \leqslant a_{0}\right\}$. Indeed, if $(t, a) \in Q_{\tau}^{3}$ with $a \leqslant a_{0}$, then from (2.9) we have

$$
p(t, a)=\pi(a)\left(\tilde{b}(t-a)+v(t-a) \int_{0}^{a} \frac{m(s)}{\pi(s)} \mathrm{d} s\right) \quad\left((t, a) \in Q_{\tau}^{3}, a \leqslant a_{0}\right),
$$

with

$$
\tilde{b}(t)=\int_{a_{b}}^{a_{\dagger}} \beta(a) p(t, a) \mathrm{d} a \quad(t \in[0, \tau]) .
$$

From (4.4), (4.7), (4.10) and (4.14) it follows that we have $\tilde{b}(t) \geqslant 0$ for every $t \in[0, \tau]$, since the density $p(t, a)$ is positive for $a \geqslant a_{b} \geqslant a_{0}$. Distinguish again the cases $v(t-a) \geqslant 0$ and $v(t-a)<0$ in (4.13) and using the positivity and the continuity of $p$ along the red and green lines represented in Figure 1 provides the positivity of the controlled solution $p$ on the subdomain $\left\{(t, a) \in Q_{\tau}^{3}, a \leqslant a_{0}\right\}$.

The case where $p_{0} \in X$ and $\tilde{p} \in \mathcal{R}$ are not necessarily continuous can be deduced from a density argument. Indeed, it suffices to note that from Remark 3.3 it follows that if $\left(p_{0, n}\right)_{n \geqslant 1} \subset X$ and $\left(\tilde{p}_{n}\right)_{n \geqslant 1} \subset \mathcal{R}$ are such that $p_{0, n} \rightarrow p_{0}$ and $\tilde{p}_{n} \rightarrow \tilde{p}$ then the corresponding state trajectories satisfy $p_{n} \rightarrow p$ in $L^{2}\left(Q_{\tau}\right)$.

Remark 4.3. The control and the state trajectory constructed in the above proof can be explicitly written in terms of $p_{0}$ and $\tilde{p}$. Indeed, applying the formulas in Remark 3.3, with $\tilde{p}-\mathbb{T}_{\tau} p_{0}$ instead 
of $\tilde{p}$ and after some calculations, it can be checked that, for almost every $(t, a) \in Q_{\tau}$ we have

$$
u(t, a)=\left\{\begin{array}{lr}
0 & \text { if } t-a<\tau-a_{\dagger}, \\
\frac{\tilde{p}(\tau+(a-t))}{\frac{\pi(\tau+(a-t))}{\tau(a-t)}-\frac{p_{0}(a-t)}{\pi(a-t)}} & \text { if } \quad \tau-a_{\dagger} \leqslant t-a \leqslant 0, \\
\frac{\tilde{p}(\tau-(t-a))-\pi(\tau-(t-a)) \eta(t-a)}{\pi(x)} \mathrm{d} x & \text { if } 0<t-a \leqslant \tau,
\end{array}\right.
$$

where

$$
\eta(s)=\int_{a_{b}}^{s+a_{\dagger}-\tau} \beta(x) \pi(x) \frac{\tilde{p}(\tau-(s-x))}{\pi(\tau-(s-x))} \mathrm{d} x+\int_{s+a_{\dagger}-\tau}^{a_{\dagger}} \beta(x) \pi(x) \frac{p_{0}(x-s)}{\pi(x-s)} \mathrm{d} x \quad(s \in[0, \tau]) .
$$

Moreover, for almost every $(t, a) \in Q_{\tau}$, we have

$$
p(t, a)= \begin{cases}\frac{\pi(a)}{\pi(a-t)} p_{0}(a-t) & \text { if } t-a<\tau-a_{\dagger}, \\ \frac{\pi(a)}{\pi(a-t)} p_{0}(a-t)\left[1-\frac{\int_{a-t}^{a} \frac{m(x)}{\pi(x)} \mathrm{d} x}{\left.\int_{a-t}^{a+(\tau-t)} \frac{m(x)}{\pi(x)} \mathrm{d} x\right]}\right. & \\ +\pi(a) \frac{\int_{a-t}^{a} \frac{m(s)}{\pi(s)} \mathrm{d} s}{\int_{a-t}^{\tau+(a-t)} \frac{m(s)}{\pi(s)} \mathrm{d} s} \frac{\tilde{p}(\tau+(a-t))}{\pi(\tau+(a-t))} & \text { if } \quad \tau-a_{\dagger} \leqslant t-a \leqslant 0, \\ \pi(a) \eta(t-a)\left[1-\frac{m(s)}{\int_{0}^{\tau-(t-a)} \frac{m(s)}{\pi(s)} \mathrm{d} s}\right] & \text { if } 0<t-a \leqslant \tau . \\ +\pi(a) \frac{\int_{0}^{a} \frac{m(x)}{\pi(x)} \mathrm{d} x}{\int_{0}^{\tau-(t-a)} \frac{m(x)}{\pi(x)} \mathrm{d} x} \frac{\tilde{p}(\tau-(t-a))}{\pi(\tau-(t-a))}\end{cases}
$$

We show below that the result in Theorem 4.2 applies for target states which are stationary solutions of (1.1), which is the case investigated in [8].

Proof of Theorem 1.1. According to Theorem 4.2, we only have to show that any solution $\tilde{p}$ of (1.2) satisfies $\tilde{p} \in \mathcal{R}$.

From [8] it follows that any solution $\tilde{p}$ of (1.2) is given by

$$
\tilde{p}(a)=C \pi(a)+\int_{0}^{a} \frac{\pi(a)}{\pi(\sigma)} m(\sigma) w(\sigma) \mathrm{d} \sigma \quad\left(a \in\left(0, a_{\dagger}\right)\right),
$$

where $C$ is determined through

$$
(1-R) C=\int_{0}^{a_{\dagger}} \beta(x) \pi(x) \int_{0}^{x} \frac{m(\sigma)}{\pi(\sigma)} w(\sigma) \mathrm{d} \sigma \mathrm{d} x
$$


and $R$ is the reproductive number of the population given by

$$
R=\int_{0}^{a_{\dagger}} \beta(a) \pi(a) \mathrm{d} a .
$$

From (4.15) it follows that for almost every $a \in\left(0, a_{\dagger}\right)$ we have

$$
\frac{\tilde{p}(a)}{\pi(a)}=C+\int_{0}^{a} \frac{m(\sigma)}{\pi(\sigma)} w(\sigma) \mathrm{d} \sigma
$$

so that $\frac{\tilde{p}}{\pi} \in L^{2}\left[0, a_{\dagger}\right]$. Moreover, for almost every $a \in\left(0, a_{\dagger}\right)$ we have from (4.15) that

$$
\begin{aligned}
\frac{\tilde{p}(a)}{\pi(a)} & -\int_{0}^{a_{\dagger}-a} \beta(x) \frac{\pi(x)}{\pi(a+x)} \tilde{p}(a+x) \mathrm{d} x \\
& =C+\int_{0}^{a} \frac{m(\sigma)}{\pi(\sigma)} w(\sigma) \mathrm{d} \sigma-\int_{0}^{a_{\dagger}-a} \beta(x) \pi(x)\left[C+\int_{0}^{a+x} \frac{m(\sigma)}{\pi(\sigma)} w(\sigma) \mathrm{d} \sigma\right] \mathrm{d} x \\
& =\int_{0}^{a} \frac{m(\sigma)}{\pi(\sigma)} w(\sigma) \mathrm{d} \sigma+C\left(1-\int_{0}^{a_{\dagger}-a} \beta(x) \pi(x) \mathrm{d} x\right)-\int_{0}^{a_{\dagger}-a} \beta(x) \pi(x) \int_{0}^{a+x} \frac{m(\sigma)}{\pi(\sigma)} w(\sigma) \mathrm{d} \sigma \mathrm{d} x .
\end{aligned}
$$

Define $\mathcal{G}:=\int_{0}^{a_{\dagger}} \beta(x) \pi(x) \int_{0}^{x} \frac{m(\sigma)}{\pi(\sigma)} w(\sigma) \mathrm{d} \sigma \mathrm{d} x$ such that from $(4.16)$ we have $(1-R) C=\mathcal{G}$. Define $r(a):=\int_{0}^{a_{\dagger}-a} \beta(x) \pi(x) \mathrm{d} x$ and $g(a):=\int_{0}^{a_{\dagger}-a} \beta(x) \pi(x) \int_{0}^{a+x} \frac{m(\sigma)}{\pi(\sigma)} w(\sigma) \mathrm{d} \sigma \mathrm{d} x$. Then (4.17) yields that for almost every $a \in\left(0, a_{\dagger}\right)$, we have

$$
\begin{aligned}
\frac{\tilde{p}(a)}{\pi(a)}-\int_{0}^{a_{\dagger}-a} & \beta(x) \frac{\pi(x)}{\pi(a+x)} \tilde{p}(a+x) \mathrm{d} x \\
& =\int_{0}^{a} \frac{m(\sigma)}{\pi(\sigma)} w(\sigma) \mathrm{d} \sigma+C(1-r(a))-g(a) \\
& =\int_{0}^{a} \frac{m(\sigma)}{\pi(\sigma)} w(\sigma) \mathrm{d} \sigma+C(R-r(a))+(\mathcal{G}-g(a)) .
\end{aligned}
$$

Let us show that the map

$$
a \mapsto \frac{\int_{0}^{a} \frac{m(\sigma)}{\pi(\sigma)} w(\sigma) \mathrm{d} \sigma+C(R-r(a))+(\mathcal{G}-g(a))}{a},
$$

is in $L^{2}\left[0, a_{\dagger}\right]$. By Hardy's inequality (see $[12, \mathrm{p} 240]$ ), the map

$$
a \mapsto \frac{\int_{0}^{a} \frac{m(\sigma)}{\pi(\sigma)} w(\sigma) \mathrm{d} \sigma}{a}
$$

lies in $L^{2}\left[0, a_{\dagger}\right]$. For the second term at the numerator in (4.19) we note that for almost every $a \in\left(0, a_{\dagger}\right)$ we have

$$
\begin{aligned}
|R-r(a)| & =\int_{a_{\dagger}-a}^{a_{\dagger}} \beta(x) \pi(x) \mathrm{d} x \\
& \leqslant a\|\beta\|_{L^{\infty}\left[0, a_{\dagger}\right]},
\end{aligned}
$$

so that the map $a \mapsto \frac{R-r(a)}{a}$ is in $L^{2}\left[0, a_{\dagger}\right]$.

We still have to tackle the third term at the numerator in (4.19). To this aim we note that for almost every $a \in\left(0, a_{\dagger}\right)$ we have

$$
\frac{\mathrm{d}}{\mathrm{d} a}(\mathcal{G}-g(a))=-\beta\left(a_{\dagger}-a\right) \pi\left(a_{\dagger}-a\right) \int_{0}^{a_{\dagger}} \frac{m(\sigma)}{\pi(\sigma)} w(\sigma) \mathrm{d} \sigma-\int_{0}^{a_{\dagger}-a} \beta(x) \pi(x) \frac{m(a+x)}{\pi(a+x)} w(a+x) \mathrm{d} x,
$$


so that the map $a \mapsto \frac{\mathrm{d}}{\mathrm{d} a}(\mathcal{G}-g(a))$ is in $L^{2}\left[0, a_{\dagger}\right]$. It follows that the map $F: a \mapsto \mathcal{G}-g(a)$ satisfies $F \in H^{1}\left[0, a_{\dagger}\right]$ with $F(0)=0$, so that from Hardy's inequality (see [12, p 240]) the map $a \mapsto \frac{F(a)}{a}$ is in $L^{2}\left[0, a_{\dagger}\right]$. Consequently, from (4.19) we have $\tilde{p} \in \mathcal{R}$.

Remark 4.4. The method employed above to prove Theorem 1.1 cannot be adapted to the case in which the state space $X=L^{2}\left[0, a_{\dagger}\right]$ is substituted by $X=L^{1}\left[0, a_{\dagger}\right]$. Indeed, if $w$ and $\tilde{p}$ are in $L^{1}$ the fact that $\tilde{p}$ is in $\mathcal{R}$ no longer holds, in general. Indeed, the map

$$
a \mapsto \frac{\int_{0}^{a} \frac{m(\sigma)}{\pi(\sigma)} w(\sigma) \mathrm{d} \sigma}{a}
$$

does not necessarily belong to $L^{1}\left[0, a_{\dagger}\right]$ for any $w \in L^{1}\left[0, a_{\dagger}\right]$ (see Hardy's inequality [12]). However, the inclusion Ran $\mathbb{T}_{\tau} \subset$ Ran $\Phi_{\tau}$ remains true for every $\tau>a_{\dagger}-a_{0}-$ see Appendix $A$.

\section{A singular perturbation problem}

In this section we study the behavior of the null controls constructed in Section 4, together with the behavior of the corresponding state trajectories, when the age interval where the control is active shrinks to the singleton $\{0\}$. Not surprisingly, we show that the null controls converge to a null control for a system in which the input appears in an additive manner in the formula giving the birth rate. The control in this limit system can be seen as an "impulse" (in the age domain) type control or, simpler, as a direct control of the birth rate. Similar singular perturbation problems for the wave or for the Schrödinger equations have been studied in Fabre [11, 9], Fabre and Puel [10] and Joly [15].

To be more precise, we consider the control system (1.1) for $a_{0}=\varepsilon \in\left(0, a_{\dagger}\right)$. According to Proposition 4.1, given $\varepsilon>0$, for every $p_{0} \in X=L^{2}\left[0, a_{\dagger}\right]$ there exists $u^{\varepsilon} \in L^{2}\left(\left[0, a_{\dagger}\right] ; X\right)$ such that the solution of

$$
\left\{\begin{array}{lr}
\dot{p}^{\varepsilon}(t, a)+\frac{\partial p^{\varepsilon}}{\partial a}(t, a)+\mu(a) p^{\varepsilon}(t, a)=\mathbb{1}_{[0, \varepsilon]}(a) u^{\varepsilon}(t, a), & (t, a) \in\left(0, a_{\dagger}\right)^{2}, \\
p^{\varepsilon}(t, 0)=\int_{0}^{a_{\dagger}} \beta(a) p^{\varepsilon}(t, a) \mathrm{d} a, & t \in\left(0, a_{\dagger}\right), \\
p^{\varepsilon}(0, a)=p_{0}(a), & a \in\left(0, a_{\dagger}\right),
\end{array}\right.
$$

satisfies

$$
p^{\varepsilon}\left(a_{\dagger}, a\right)=0 \quad\left(a \in\left(0, a_{\dagger}\right)\right) .
$$

Moreover, by a slight variation of the calculations used to derive the formula in Remark 4.3, it is easy to check that the control $u^{\varepsilon}$ defined by

$$
u^{\varepsilon}(t, a)= \begin{cases}-\frac{\int_{t-a}^{a_{\dagger}} \beta(x) \pi(x) \frac{p_{0}(x-(t-a))}{\pi(x-(t-a))} \mathrm{d} x}{\int_{0}^{a_{\dagger}-(t-a)} \frac{\mathbb{1}_{[0, \varepsilon]}(x)}{\pi(x)} \mathrm{d} x} & \left((t, a) \in\left(0, a_{\dagger}\right)^{2}, t-a \geqslant 0\right), \\ 0 & \left((t, a) \in\left(0, a_{\dagger}\right)^{2}, t-a<0\right),\end{cases}
$$

satisfies the above conditions. With $u_{\varepsilon}$ defined by (5.3), the controlled density $p^{\varepsilon}$ solution of (5.1) is given by

$$
p^{\varepsilon}(t, a)=\left\{\begin{array}{lr}
\frac{\pi(a)}{\pi(a-t)} p_{0}(a-t) & \text { if } t-a<0, \\
\pi(a) \eta(t-a)\left[1-\frac{\int_{0}^{a} \frac{\mathbb{1}_{[0, \varepsilon]}(x)}{\pi(x)} \mathrm{d} x}{\int_{0}^{a_{\dagger}-(t-a)} \frac{\mathbb{1}_{[0, \varepsilon]}(x)}{\pi(x)} \mathrm{d} x}\right] & \text { if } 0<t-a \leqslant a_{\dagger},
\end{array}\right.
$$


where

$$
\eta(s)=\int_{s}^{a_{\dagger}} \beta(x) \pi(x) \frac{p_{0}(x-s)}{\pi(x-s)} \mathrm{d} x \quad\left(s \in\left[0, a_{\dagger}\right]\right) .
$$

We also consider the following boundary controlled system, already studied in [8]:

$$
\left\{\begin{array}{lrl}
\dot{p}(t, a)+\frac{\partial p}{\partial a}(t, a)+\mu(a) p(t, a)=0, & (t, a) \in\left(0, a_{\dagger}\right)^{2}, \\
p(t, 0)=\int_{0}^{a_{\dagger}} \beta(a) p(t, a) \mathrm{d} a+v(t), & t \in\left(0, a_{\dagger}\right), \\
p(0, a)=p_{0}(a), & a \in\left(0, a_{\dagger}\right),
\end{array}\right.
$$

where $v \in L^{2}\left(\left[0, a_{\dagger}\right]\right)$. Using a recursive argument as in the proof of Theorem 3.2, it can be easily shown that taking

$$
v(t):=-\int_{t}^{a_{\dagger}} \beta(a) \frac{\pi(a)}{\pi(a-t)} p_{0}(a-t) \mathrm{d} a \quad\left(t \in\left[0, a_{\dagger}\right]\right),
$$

the solution $p$ of (5.5) satisfies

$$
p\left(a_{\dagger}, a\right)=0 \quad\left(a \in\left(0, a_{\dagger}\right)\right) .
$$

More precisely, with $v$ defined by (5.6), the controlled density $p$ solution of (5.5) is given by

$$
p(t, a)= \begin{cases}\frac{\pi(a)}{\pi(a-t)} p_{0}(a-t) & \text { if } t \leqslant a \\ 0 & \text { if } t>a .\end{cases}
$$

The main result of this section is:

Theorem 5.1. Given $p_{0} \in L^{2}\left(\left[0, a_{\dagger}\right]\right)$, consider the family of distributed controls $\left(u^{\varepsilon}\right)_{\varepsilon \in\left(0, a_{\dagger}\right)}$ in $L^{2}\left(\left[0, a_{\dagger}\right] ; L^{2}\left(\left[0, a_{\dagger}\right]\right)\right)$ and the boundary control $v$ in $L^{2}\left[0, a_{\dagger}\right]$ respectively defined by $(5.3)$ and $(5.6)$. Then we have

1. $\lim _{\varepsilon \rightarrow 0^{+}} u^{\varepsilon} \mathbb{1}_{[0, \varepsilon]}=v \delta_{0}$ weakly in $L^{2}\left(\left[0, a_{\dagger}\right], \mathcal{D}\left(A^{*}\right)^{\prime}\right)$, where $\delta_{0}$ stands for the Dirac mass concentrated at the origin;

2.

$$
\lim _{\varepsilon \rightarrow 0^{+}}\left\|p^{\varepsilon}-p\right\|_{C\left(\left[0, a_{\dagger}\right] ; L^{2}\left(\left[0, a_{\dagger}\right]\right)\right)}=0,
$$

where $p$ is the solution of (5.5).

Proof. Let $\varepsilon \in\left(0, a_{\dagger} / 2\right)$. It is easy to check that every function in $\mathcal{D}\left(A^{*}\right)$ belongs to $H^{1}(0, a *)$ for every $a * \in\left(0, a_{\dagger} *\right)$. In particular, for every $\varphi \in L^{2}\left(\left[0, a_{\dagger}\right], \mathcal{D}\left(A^{*}\right)\right)$ and every $t \in\left[0, a_{\dagger}\right]$, we have $\varphi(t, \cdot) \in L^{\infty}\left(0, a_{\dagger} / 2\right)$.

We introduce the following notations:

$$
f(t, a):= \begin{cases}-\int_{t-a}^{a_{\dagger}} \beta(x) \pi(x) \frac{p_{0}(x-(t-a))}{\pi(x-(t-a))} \mathrm{d} x & \left((t, a) \in\left(0, a_{\dagger}\right)^{2}, t-a \geqslant 0\right), \\ 0 & \left((t, a) \in\left(0, a_{\dagger}\right)^{2}, t-a<0\right),\end{cases}
$$

and

$$
k^{\varepsilon}(t, a):=\int_{0}^{a_{\dagger}-(t-a)} \frac{\mathbb{1}_{[0, \varepsilon]}(x)}{\pi(x)} \mathrm{d} x \quad\left((t, a) \in\left(0, a_{\dagger}\right)^{2}\right),
$$

so that from (5.3), (5.9) and (5.10) we have

$$
u^{\varepsilon}(t, a)=\frac{f(t, a)}{k^{\varepsilon}(t, a)} \quad\left((t, a) \in\left(0, a_{\dagger}\right)^{2}\right) .
$$


Let $\varphi \in L^{2}\left(\left[0, a_{\dagger}\right], \mathcal{D}\left(A^{*}\right)\right)$, and denote by

$$
g_{\varepsilon}(t):=\int_{0}^{a_{\dagger}} \mathbb{1}_{[0, \varepsilon]}(a) u^{\varepsilon}(t, a) \varphi(t, a) \mathrm{d} a=\int_{0}^{\varepsilon} \frac{f(t, a)}{k^{\varepsilon}(t, a)} \varphi(t, a) \mathrm{d} a \quad\left(t \in\left[0, a_{\dagger}\right]\right) .
$$

The aim is to show that $\lim _{\varepsilon \rightarrow 0} g_{\varepsilon}=v \varphi(\cdot, 0)$ in $L^{2}\left[0, a_{\dagger}\right]$. To this aim, we use the Lebesgue's Dominated Convergence Theorem. Firstly, let us show that for every $t \in\left[0, a_{\dagger}\right]$ we have the following pointwise convergence:

$$
\lim _{\varepsilon \rightarrow 0^{+}} g_{\varepsilon}(t)=v(t) \varphi(t, 0) \quad\left(t \in\left[0, a_{\dagger}\right]\right) .
$$

For every $t \in\left[0, a_{\dagger}\right)$, take $\varepsilon<a_{\dagger}-t$ small enough so that $\varepsilon<a_{\dagger}-(t-a)$ for every $a \in(0, \varepsilon)$. It follows from (5.10) that we have

$$
k^{\varepsilon}(t, a)=\int_{0}^{\varepsilon} \frac{\mathrm{d} x}{\pi(x)} \quad(a \in(0, \varepsilon)),
$$

so that from (5.11) and (5.13) we have

$$
g_{\varepsilon}(t)=\frac{1}{\int_{0}^{\varepsilon} \frac{1}{\pi(x)} \mathrm{d} x} \int_{0}^{\varepsilon} f(t, a) \varphi(t, a) \mathrm{d} a,
$$

and, using the last formula and the fact that $f(t, 0)=v(t)$, we have

$$
\lim _{\varepsilon \rightarrow 0} g_{\varepsilon}(t)=v(t) \varphi(t, 0) \quad\left(t \in \left[0, a_{\dagger}[) .\right.\right.
$$

For $t=a_{\dagger}$, it follows from (5.10) that we have

$$
k^{\varepsilon}\left(a_{\dagger}, a\right)=\int_{0}^{a} \frac{\mathrm{d} x}{\pi(x)} \quad(a \in(0, \varepsilon)),
$$

so that from (5.11) and (5.15) we have

$$
g_{\varepsilon}\left(a_{\dagger}\right)=\int_{0}^{\varepsilon} \frac{f\left(a_{\dagger}, a\right)}{\int_{0}^{a} \frac{1}{\pi(x)} \mathrm{d} x} \varphi\left(a_{\dagger}, a\right) \mathrm{d} a,
$$

and, using the above formula, together with the fact that $v\left(a_{\dagger}\right)=0$, we obtain that

$$
\lim _{\varepsilon \rightarrow 0} g_{\varepsilon}\left(a_{\dagger}\right)=v\left(a_{\dagger}\right) \varphi\left(a_{\dagger}, 0\right),
$$

Consequently, it follows from (5.14) and (5.16) that the pointwise convergence (5.12) holds for every $t \in\left[0, a_{\dagger}\right]$.

Secondly, let us find a function $h \in L^{2}\left[0, a_{\dagger}\right]$ such that

$$
\left|g_{\varepsilon}(t)\right| \leqslant h(t) \quad\left(\varepsilon \in\left(0, a_{\dagger} / 2\right), t \in\left[0, a_{\dagger}\right]\right) .
$$

It is easy to check that for every $t \in\left[0, a_{\dagger}-\varepsilon\right]$ we have

$$
\left.k^{\varepsilon}(t, a)=\int_{0}^{\varepsilon} \frac{\mathrm{d} x}{\pi(x)} \quad \text { if } a \in(0, \varepsilon)\right),
$$

and for every $t \in\left[a_{\dagger}-\varepsilon, a_{\dagger}\right]$ we have

$$
k^{\varepsilon}(t, a)= \begin{cases}\int_{0}^{a_{\dagger}-(t-a)} \frac{\mathrm{d} x}{\pi(x)} & \text { if } a \in\left(0, \varepsilon+t-a_{\dagger}\right), \\ \int_{0}^{\varepsilon} \frac{\mathrm{d} x}{\pi(x)} & \text { if } a \in\left(\varepsilon+t-a_{\dagger}, \varepsilon\right) .\end{cases}
$$


It follows from (5.11), (5.18) and (5.19) that we have

$$
g_{\varepsilon}(t)= \begin{cases}\frac{1}{\int_{0}^{\varepsilon} \frac{1}{\pi(x)} \mathrm{d} x} \int_{0}^{\varepsilon} f(t, a) \varphi(t, a) \mathrm{d} a, & \left(t \in\left[0, a_{\dagger}-\varepsilon\right]\right), \\ \int_{0}^{\varepsilon+t-a_{\dagger}} \frac{f(t, a) \varphi(t, a)}{\int_{0}^{a_{\dagger}-(t-a)} \frac{1}{\pi(x)} \mathrm{d} x} \mathrm{~d} a+\frac{1}{\int_{0}^{\varepsilon} \frac{1}{\pi(x)} \mathrm{d} x} \int_{\varepsilon+t-a_{\dagger}}^{\varepsilon} f(t, a) \varphi(t, a) \mathrm{d} a, & \left(t \in\left[a_{\dagger}-\varepsilon, a_{\dagger}\right]\right) .\end{cases}
$$

For every $t \in\left[0, a_{\dagger}-\varepsilon\right]$, since $f \in L^{\infty}\left(\left[0, a_{\dagger}\right]^{2}\right)$ and $\varphi(t, \cdot) \in L^{\infty}\left(\left[0, a_{\dagger} / 2\right]\right)$, from (5.20) we get

$$
\left|g_{\varepsilon}(t)\right| \leqslant\|f\|_{L^{\infty}\left(\left[0, a_{\dagger}\right]^{2}\right)}\|\varphi(t, \cdot)\|_{L^{\infty}\left(\left[0, a_{\dagger} / 2\right]\right)} .
$$

For every $t \in\left[a_{\dagger}-\varepsilon, a_{\dagger}\right]$, from (5.20) we have

$$
\left|g_{\varepsilon}(t)\right| \leqslant \int_{0}^{\varepsilon} \frac{f(t, a) \varphi(t, a)}{a_{\dagger}-(t-a)} \mathrm{d} a+\frac{1}{\varepsilon} \int_{0}^{\varepsilon} f(t, a) \varphi(t, a) \mathrm{d} a .
$$

Since $p_{0} \in L^{2}\left[0, a_{\dagger}\right]$, Cauchy-Schwartz inequality gives

$$
|f(t, a)| \leqslant K\left\|p_{0}\right\|_{L^{2}\left(0, a_{\dagger}\right)} \sqrt{a_{\dagger}-(t-a)},
$$

for some constant $K \geqslant 0$. It follows from (5.22) and (5.23) that we have

$$
\left|g_{\varepsilon}(t)\right| \leqslant K\left\|p_{0}\right\|_{L^{2}\left(0, a_{\dagger}\right)}\|\varphi(t, \cdot)\|_{L^{\infty}\left(\left[0, a_{\dagger} / 2\right]\right)} \int_{0}^{a_{\dagger} / 2} \frac{\mathrm{d} a}{\sqrt{a_{\dagger}-(t-a)}}+\|f\|_{L^{\infty}\left(\left[0, a_{\dagger}\right]^{2}\right)}\|\varphi(t, \cdot)\|_{L^{\infty}\left(\left[0, a_{\dagger} / 2\right]\right)},
$$

and the following majoration holds:

$$
\left|g_{\varepsilon}(t)\right| \leqslant 2 K\left\|p_{0}\right\|_{L^{2}\left(0, a_{\dagger}\right)}\|\varphi(t, \cdot)\|_{L^{\infty}\left(\left[0, a_{\dagger} / 2\right]\right)} \sqrt{3 a_{\dagger} / 2}+\|f\|_{L^{\infty}\left(\left[0, a_{\dagger}\right]^{2}\right)}\|\varphi(t, \cdot)\|_{L^{\infty}\left(\left[0, a_{\dagger} / 2\right]\right)} .
$$

Define, for every $t \in\left[0, a_{\dagger}\right]$,

$$
h(t):=2 K\left\|p_{0}\right\|_{L^{2}\left(0, a_{\dagger}\right)}\|\varphi(t, \cdot)\|_{L^{\infty}\left(\left[0, a_{\dagger} / 2\right]\right)} \sqrt{3 a_{\dagger} / 2}+\|f\|_{L^{\infty}\left(\left[0, a_{\dagger}\right]^{2}\right)}\|\varphi(t, \cdot)\|_{L^{\infty}\left(\left[0, a_{\dagger} / 2\right]\right)} .
$$

It follows from (5.21) and (5.25) that for every $\varepsilon \in\left(0, a_{\dagger} / 2\right)$ and every $t \in\left[0, a_{\dagger}\right]$, we have

$$
\left|g_{\varepsilon}(t)\right| \leqslant h(t) \quad\left(\varepsilon \in\left(0, a_{\dagger} / 2\right), t \in\left[0, a_{\dagger}\right]\right),
$$

with $h \in L^{2}\left(\left[0, a_{\dagger}\right]\right)$. The pointwise convergence (5.12) and the above domination (5.27) gives

$$
\lim _{\varepsilon \rightarrow 0} g_{\varepsilon}=v \varphi(\cdot, 0) \text { in } L^{2}\left[0, a_{\dagger}\right],
$$

so that we have

$$
\lim _{\varepsilon \rightarrow 0^{+}} u^{\varepsilon} \mathbb{1}_{[0, \varepsilon]}=v \delta_{0} \text { weakly in } L^{2}\left(\left[0, a_{\dagger}\right], \mathcal{D}\left(A^{*}\right)^{\prime}\right) .
$$

Concerning the convergence of the state trajectories, it follows from (5.4) and (5.8) that for every $t \in\left[0, a_{\dagger}\right]$,

$$
\begin{aligned}
\int_{0}^{a_{\dagger}}\left|p^{\varepsilon}(t, a)-p(t, a)\right|^{2} \mathrm{~d} a & \leqslant\|\eta\|_{L^{\infty}\left[0, a_{\dagger}\right]} \int_{0}^{\varepsilon}\left(1-\frac{\int_{0}^{a} \frac{\mathbb{1}_{[0, \varepsilon]}(x)}{\pi(x)} \mathrm{d} x}{\int_{0}^{a_{\dagger}-(t-a)} \frac{\mathbb{1}_{[0, \varepsilon]}(x)}{\pi(x)} \mathrm{d} x}\right)^{2} \mathrm{~d} a \\
& \leqslant\|\eta\|_{L^{\infty}\left[0, a_{\dagger}\right]} \varepsilon .
\end{aligned}
$$

The convergence stated in Theorem (5.1) easily follows from (5.30). 


\section{Control of an infection-age model}

The methodology developed in the previous sections is essentially applicable to linear systems. However, the fact that controls are explicitly determined by calculations along characteristics, allows, in some particular, situations to tackle nonlinear systems. The aim of this section is thus to provide an example of application of our methods for a nonlinear controlled infection-age model which writes:

$$
\left\{\begin{array}{l}
\frac{\mathrm{d} S}{\mathrm{~d} t}(t)=-S(t) \int_{0}^{a_{\dagger}} \beta(a) i(t, a) \mathrm{d} a \\
\frac{\partial i}{\partial t}(t, a)+\frac{\partial i}{\partial a}(t, a)=-\mu(a) i(t, a)-m(a) u(t, a) \\
i(t, 0)=S(t) \int_{0}^{a_{\dagger}} \beta(a) i(t, a) \mathrm{d} a
\end{array}\right.
$$

with the initial condition

$$
S(0)=S_{0} \geqslant 0 \text { and } i(0, \cdot)=i_{0} \in L_{+}^{2}\left[0, a_{\dagger}\right] .
$$

In the model (6.1), the population is decomposed into the class (S) of susceptible individuals and the class (I) of infected individuals. The number of individuals in the class (S) at time $t$ is $S(t)$. The age of infection $a \in\left[0, a_{\dagger}\right]$ is the time since the infection began (where $a_{\dagger}$ denotes the maximum age of the epidemic), and $i(t, a)$ is the density of infected individuals with respect to the age infection. That is to say that for two given age values $a_{1}, a_{2}: 0 \leqslant a_{1}<a_{2} \leqslant a_{\dagger}$ the number of infected individuals with age of infection $a$ between $a_{1}$ and $a_{2}$ is

$$
\int_{a_{1}}^{a_{2}} i(t, a) \mathrm{d} a .
$$

The function $\beta(a)$ is the force of infection at the age of infection $a$. We assume that there exists $a_{b} \in\left(0, a_{\dagger}\right)$ such that

$$
\beta(a)=0 \quad\left(a \in\left(0, a_{b}\right) \text { a.e. }\right)
$$

that is to say we interpret infection age to an exposed period (infected, but not yet infectious) from $a=0$ to $a=a_{b}$ and an infectious period from $a=a_{b}$ to $a=a_{\dagger}$. The quantity

$$
\int_{0}^{a_{\dagger}} \beta(a) i(t, a) \mathrm{d} a
$$

is the number of infectious individuals within the subpopulation (I). Finally, $\mu(a)$ is the exit (or (and) mortality or (and) recovery) rate of infected individuals with an age of infection $a \geqslant 0$. As a consequence the quantity

$$
\pi(a):=\exp \left(-\int_{0}^{a} \mu(\sigma) \mathrm{d} \sigma\right)
$$

is the probability for an individual to stay in the class (I) after a period of time $a \geqslant 0$.

System (6.1) can be seen as a semilinear Cauchy problem in an appropriate Banch space, in which the closed linear operator is not densely defined (see, for instance, Magal and Ruan [19, 20, 21]. In the above references, the existence and the uniqueness of solutions, for a given $L^{2}$ input function $u$, is proved by using a step by step procedure which preserves the positivity of solutions. The global in time existence and uniqueness follows since no finite time blowup occurs. Here, we aim to find a control function $u$ such as the density of infected individuals in the age-infection model (6.1) becomes null in finite time. We suppose that $m=\mathbb{1}_{\left[0, a_{0}\right]}$ is the characteristic function of the interval $\left(0, a_{0}\right)$, with $a_{0} \leqslant a_{b}$ : this means that we are able to control infected individuals that are still not infectious. The below proposition show that by controlling the infected individuals (i.e. by using quarantine) before their infectiousness period we can eradicate the epidemic in finite time. 
Proposition 6.1. Let assumption $(6.2)$ be satisfied. Let $i_{0} \in L^{2}\left[0, a_{\dagger}\right]$ with $i_{0}(a) \geqslant 0$ for almost every $a \in\left(0, a_{\dagger}\right)$ and let $S_{0} \geqslant 0$. Then for every $\tau>a_{\dagger}-a_{0}$, there exists a control $u \in L^{2}\left([0, \tau] ; L^{2}\left[0, a_{\dagger}\right]\right)$ such that the controlled density of infected individuals $i$ given by (6.1) satisfies

$$
i(\tau, a)=0 \quad\left(a \in\left(0, a_{\dagger}\right) \text { a.e. }\right) .
$$

Moreover the controlled density of infected individuals remains positive that is to say

$$
i(t, a) \geqslant 0 \text { a.e. }(t, a) \in Q_{\tau} .
$$

By a slight variation of the calculations in Section 2 we can check the solution of (6.1) satisfies:

$$
S(t)=S_{0} \exp \left(-\int_{0}^{t} \int_{0}^{a_{\dagger}} \beta(a) i(s, a) \mathrm{d} a \mathrm{~d} s\right),
$$

and

$$
i(t, a):= \begin{cases}\frac{\pi(a)}{\pi(a-t)} i_{0}(a-t)+c(t, a), & t \leqslant a, \\ \pi(a) \tilde{b}(t-a)+c(t, a), & a<t,\end{cases}
$$

where

$$
c(t, a):= \begin{cases}\int_{a-t}^{a} \frac{\pi(a)}{\pi(s)} m(s) u(s-(a-t), s) \mathrm{d} s, & t \leqslant a, \\ \int_{0}^{a} \frac{\pi(a)}{\pi(s)} m(s) u(s+(t-a), s) \mathrm{d} s, & a<t,\end{cases}
$$

and $t \mapsto \tilde{b}(t)$ is the unique continuous function satisfying the Volterra integral equation

$$
\begin{aligned}
\tilde{b}(t)=S(t)\left\{\int_{0}^{\min \left(t, a_{\dagger}\right)} \beta(a)[\pi(a) \tilde{b}(t\right. & -a)+c(t, a)] \mathrm{d} a \\
& \left.+\int_{\min \left(t, a_{\dagger}\right)}^{a_{\dagger}} \beta(a)\left[\frac{\pi(a)}{\pi(a-t)} i_{0}(a-t)+c(t, a)\right] \mathrm{d} a\right\} .
\end{aligned}
$$

Proof of Proposition 6.1. The remark suggesting the approach below is that, assuming that $S$ is a given function, the construction of the control in Theorem 4.2 and, more precisely, in Remark 4.3 , adapts to the controllability of the system formed by the last two equations in (6.1). This motivates the below construction of the control function for the system (6.1).

As previously, we focus on the case where $\tau \in] a_{\dagger}-a_{0}, a_{\dagger}[$.

Case $t-a<0$ : We first set

$$
u(t, a)= \begin{cases}0 & \text { if } t-a<\tau-a_{\dagger}, \\ \frac{i_{0}(a-t)}{\pi(a-t)} & \text { if } \tau-a_{\dagger} \leqslant t-a \leqslant 0, \\ \int_{a-t}^{\tau+(a-t)} \frac{m(x)}{\pi(x)} \mathrm{d} x & \end{cases}
$$

so that from (6.4) and (6.7) we have

$$
i(t, a)=\left\{\begin{array}{lr}
\frac{\pi(a)}{\pi(a-t)} i_{0}(a-t) & \text { if } t-a<\tau-a_{\dagger}, \\
\frac{\pi(a)}{\pi(a-t)} i_{0}(a-t)\left[1-\frac{\int_{a-t}^{a} \frac{m(x)}{\pi(x)} \mathrm{d} x}{\int_{a-t}^{a+(\tau-t)} \frac{m(x)}{\pi(x)} \mathrm{d} x}\right] & \text { if } \tau-a_{\dagger} \leqslant t-a \leqslant 0 .
\end{array}\right.
$$


The above formula (6.8) clearly implies that

$$
i(\tau, a)=0 \quad\left(a \in\left(\tau, a_{\dagger}\right) \text { a.e. }\right) .
$$

Case $0<t-a<a_{b}$ : using (6.8), we are able to compute $S(t)$ for every $t \in\left[0, a_{b}\right]$. Indeed, after an easy calculation using (6.8) it follows that for every $t \in\left[0, a_{b}\right]$,

$$
\int_{0}^{a_{\dagger}} \beta(a) i(t, a) \mathrm{d} a=\int_{a_{b}}^{a_{\dagger}} \beta(a) i(t, a) \mathrm{d} a=\int_{t+a_{\dagger}-\tau}^{a_{\dagger}} \beta(a) \frac{\pi(a)}{\pi(a-t)} i_{0}(a-t) \mathrm{d} a .
$$

In the sequel, we set

$$
f(t):=\int_{t+a_{\dagger}-\tau}^{a_{\dagger}} \beta(a) \frac{\pi(a)}{\pi(a-t)} i_{0}(a-t) \mathrm{d} a \quad(t \in[0, \tau]),
$$

so that using (6.3), (6.10) and (6.11) we have

$$
S(t)=S_{0} e^{-\int_{0}^{t} f(s) \mathrm{d} s} \quad\left(t \in\left[0, a_{b}\right]\right) .
$$

Then for every $t \in\left[0, a_{b}\right]$, using (6.2), (6.6), (6.10) and (6.12) we have

$$
\tilde{b}(t)=S(t) \int_{a_{b}}^{a_{\dagger}} \beta(a) i(t, a) \mathrm{d} a=S_{0} f(t) e^{-\int_{0}^{t} f(s) \mathrm{d} s} \quad\left(t \in\left[0, a_{b}\right]\right) .
$$

Denoting by

$$
\chi(t):=S_{0} f(t) e^{-\int_{0}^{t} f(s) \mathrm{d} s} \quad(t \in[0, \tau]),
$$

it follows from $(6.13)$ and $(6.14)$ that we have $\tilde{b}(t)=\chi(t)$ for every $t \in\left[0, a_{b}\right]$. Then we set for almost every $(t, a) \in Q_{\tau}$ with $0<t-a \leqslant a_{b}$ :

$$
u(t, a)=\frac{\chi(t-a)}{\int_{0}^{\tau-(t-a)} \frac{m(x)}{\pi(x)} \mathrm{d} x}
$$

so that from (6.4) we have

$$
i(t, a)=\pi(a) \chi(t-a)\left[1-\frac{\int_{0}^{a} \frac{m(s)}{\pi(s)} \mathrm{d} s}{\int_{0}^{\tau-(t-a)} \frac{m(s)}{\pi(s)} \mathrm{d} s}\right] \text { if } 0<t-a \leqslant a_{b} .
$$

It follows from (6.16) that we have

$$
i(\tau, a)=0 \quad\left(a \in\left(\tau-a_{b}, \tau\right) \text { a.e. }\right) .
$$

Case $j a_{b}<t-a<(j-1) a_{b}$ for some integer $j \geqslant 1$ : The above calculations suggest as possible control driving the initial state to $u(t, a)$ where

$$
u(t, a)=\left\{\begin{array}{lc}
0 & \text { if } t-a<\tau-a_{\dagger}, \\
\frac{\frac{i_{0}(a-t)}{\pi(a-t)}}{\int_{a-t}^{\tau+(a-t)} \frac{m(x)}{\pi(x)} \mathrm{d} x} \text { if } & \tau-a_{\dagger} \leqslant t-a \leqslant 0, \\
\frac{\chi(t-a)}{\int_{0}^{\tau-(t-a)} \frac{m(x)}{\pi(x)} \mathrm{d} x} & \text { if } 0<t-a \leqslant \tau,
\end{array}\right.
$$


where $\chi$ is defined by (6.14). With $u$ defined by (6.18), it can be checked using a recursive argument as in the proof of Theorem 3.2 that we have

$$
S(t)=S_{0} e^{-\int_{0}^{t} f(s) \mathrm{d} s} \quad\left(t \in\left[j a_{b},(j+1) a_{b}\right]\right),
$$

which implies that

$$
\tilde{b}(t)=\chi(t) \quad\left(t \in\left[j a_{b},(j+1) a_{b}\right]\right),
$$

so that using $(6.4),(6.18)$ and $(6.20)$ we get

$$
i(t, a)=\pi(a) \chi(t-a)\left[1-\frac{\int_{0}^{a} \frac{m(s)}{\pi(s)} \mathrm{d} s}{\int_{0}^{\tau-(t-a)} \frac{m(s)}{\pi(s)} \mathrm{d} s}\right] \text { if } j a_{b}<t-a \leqslant(j+1) a_{b},
$$

and it follows from the above formula (6.21) that we have

$$
i(\tau, a)=0 \quad\left(a \in\left[\tau-(j+1) a_{b}, \tau-j a_{b}\right] \quad \text { a.e. }\right) .
$$

Finally, with $u$ defined by (6.18) we have

$$
i(t, a)= \begin{cases}\frac{\pi(a)}{\pi(a-t)} i_{0}(a-t) & \text { if } t-a<\tau-a_{\dagger}, \\ \frac{\pi(a)}{\pi(a-t)} i_{0}(a-t)\left[1-\frac{\int_{a-t}^{a} \frac{m(x)}{\pi(x)} \mathrm{d} x}{\left.\int_{a-t}^{a+(\tau-t)} \frac{m(x)}{\pi(x)} \mathrm{d} x\right]}\right. & \text { if } \tau-a_{\dagger} \leqslant t-a \leqslant 0, \\ \pi(a) \chi(t-a)\left[1-\frac{\int_{0}^{a} \frac{m(s)}{\pi(s)} \mathrm{d} s}{\int_{0}^{\tau-(t-a)} \frac{m(s)}{\pi(s)} \mathrm{d} s}\right] & \text { if } 0<t-a \leqslant \tau,\end{cases}
$$

where we recall that we have defined for every $t \in[0, \tau]$ :

$$
\chi(t):=S_{0} f(t) e^{-\int_{0}^{t} f(s) \mathrm{d} s},
$$

so that the positivity of the controlled density $i$ is clear. Moreover, it can be checked that we have $u \in L^{2}\left([0, \tau] ; L^{2}\left[0, a_{\dagger}\right]\right)$ thanks to Hardy's inequality [12].

\section{A Null-controllability in $L^{1}\left[0, a_{\dagger}\right]$.}

In this Appendix, we investigate the null controllability of system (1.1) when the state space $X$ is chosen to be $L^{1}\left[0, a_{\dagger}\right]$ instead of $L^{2}\left[0, a_{\dagger}\right]$.

Proposition A.1. For $\tau>0$ we denote by $\left(\mathbb{T}_{\tau}\right)_{\tau>0}$ and $\left(\Phi_{\tau}\right)_{\tau>0}$ the semigroup acting on $L^{1}\left[0, a_{\dagger}\right]$ and the control to state maps, defined on $L^{1}[0, \tau]$ with values in $L^{1}\left[0, a_{\dagger}\right]$, defined according to Remark (2.2). Then for every $\tau>a_{\dagger}-a_{0}$ we have $\operatorname{Ran} \mathbb{T}_{\tau} \subset \operatorname{Ran} \Phi_{\tau}$, i.e., the system (1.1), with state space $X=L^{1}\left[0, a_{\dagger}\right]$ and input space $U=L^{1}[0, \tau]$ is null-controllable in any time $\tau>a_{\dagger}-a_{0}$.

Proof. We can assume, without loss of generality, that $a_{0} \leqslant a_{b}$. Let $\tau \in\left(a_{\dagger}-a_{0}, a_{\dagger}\right)$. Given $p_{0} \in X$, we look for a control $u \in L^{1}\left([0, \tau] ; L^{1}\left[0, a_{\dagger}\right]\right)$ such that the solution $p$ of (1.1) satisfies 
$p(\tau, \cdot)=0$. To this aim, we use the control constructed in Remark 3.3 (with $\tilde{p}=0$ ) with a slight modification (see Figure 2 for an illustration of the modification). Let $\delta \in\left(a_{\dagger}-a_{0}, \tau\right)$. We set

$$
u(t, a)=\left\{\begin{array}{lr}
0 & \text { if } t-a<\delta-a_{\dagger}, \\
-\frac{\frac{p_{0}(a-t)}{\pi(a-t)}}{\int_{a-t}^{\tau+(a-t)} \frac{m(x)}{\pi(x)} \mathrm{d} x} \text { if } \delta-a_{\dagger} \leqslant t-a \leqslant 0, \\
-\frac{\eta(t-a)}{\int_{0}^{\tau-(t-a)} \frac{m(x)}{\pi(x)} \mathrm{d} x} & \text { if } 0<t-a \leqslant \delta, \\
0 & \text { if } \delta<t-a \leqslant \tau,
\end{array}\right.
$$

where

$$
\eta(s)=\int_{s+a_{\dagger}-\delta}^{a_{\dagger}} \beta(x) \pi(x) \frac{p_{0}(x-s)}{\pi(x-s)} \mathrm{d} x \quad(s \in[0, \delta]) .
$$

With $u$ defined by (1.23), it can be checked using a recursive argument as in the proof of Theorem 3.2 that we have

$$
p(t, a)= \begin{cases}\frac{\pi(a)}{\pi(a-t)} p_{0}(a-t) & \text { if } t-a<\delta-a_{\dagger}, \\ \frac{\pi(a)}{\pi(a-t)} p_{0}(a-t)\left[1-\frac{\int_{a-t}^{a} \frac{m(x)}{\pi(x)} \mathrm{d} x}{\left.\int_{a-t}^{a+(\tau-t)} \frac{m(x)}{\pi(x)} \mathrm{d} x\right]}\right. & \text { if } \delta-a_{\dagger} \leqslant t-a \leqslant 0, \\ \pi(a) \eta(t-a)\left[1-\frac{\int_{0}^{a} \frac{m(s)}{\pi(s)} \mathrm{d} s}{\int_{0}^{\tau-(t-a)} \frac{m(s)}{\pi(s)} \mathrm{d} s}\right] & \text { if } 0<t-a \leqslant \delta .\end{cases}
$$

It follows from the above formula (1.24) that we have

$$
p(\tau, a)=0 \quad\left(a \in\left[a_{\dagger}-\delta, a_{\dagger}\right] \quad \text { a.e. }\right) .
$$

If $t \in\left[\delta, a_{\dagger}\right]$, it follows from (1.24) that we have

$$
\tilde{b}(t)=\int_{a_{b}}^{a_{\dagger}} \beta(a) p(t, a) \mathrm{d} a=0,
$$

so that from (2.9) and (1.23) we get

$$
p(t, a)=0 \quad(\delta<t-a \leqslant \tau) .
$$




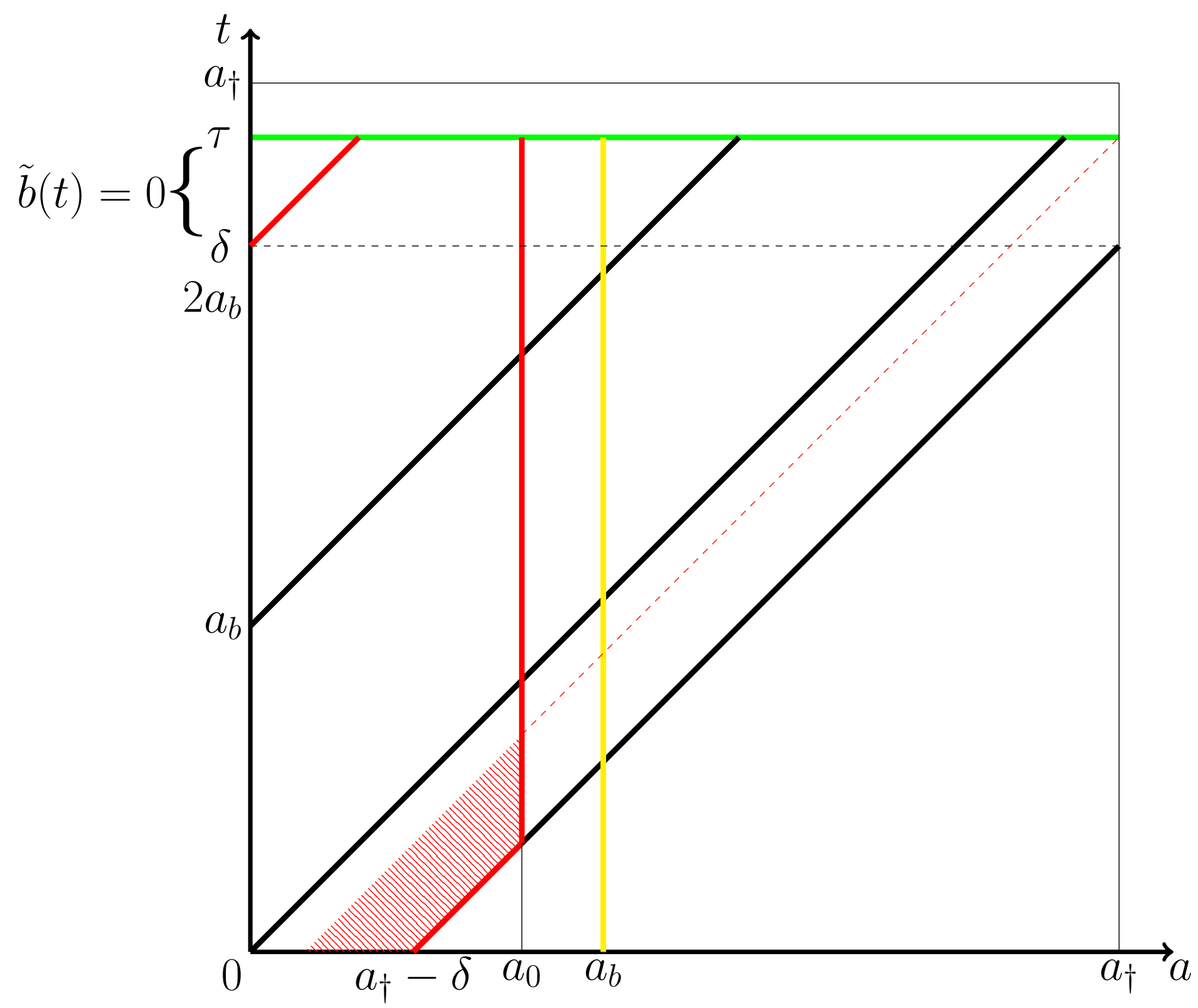

Figure 2: In this Figure, the control acts in the subset of $Q_{\tau}=[0, \tau] \times\left[0, a_{\dagger}\right]$ delimited by the line $a=a_{0}$ and the red curve. The hatched region corresponds to the slight modification of the control region (compared to the control pictured in Figure 1). Due to this modification, the value of $\tilde{b}(t)=\int_{0}^{a_{\dagger}} \beta(a) p(t, a) \mathrm{d} a$ is null for every $t \in\left[\delta, a_{\dagger}\right]$, and we can choose $u(t, a)=0$ for $\delta \leqslant t-a \leqslant a_{\dagger}$ to avoid the singularity in the neighborhood of the point $(\tau, 0)$.

It follows from (1.24) and (1.26) that the controlled density of population $p$ is given by

$$
p(t, a)= \begin{cases}\frac{\pi(a)}{\pi(a-t)} p_{0}(a-t) & \text { if } t-a<\delta-a_{\dagger}, \\ \frac{\pi(a)}{\pi(a-t)} p_{0}(a-t)\left[1-\frac{\int_{a-t}^{a} \frac{m(x)}{\pi(x)} \mathrm{d} x}{\left.\int_{a-t}^{a+(\tau-t)} \frac{m(x)}{\pi(x)} \mathrm{d} x\right]}\right. & \text { if } \delta-a_{\dagger} \leqslant t-a \leqslant 0, \\ \pi(a) \eta(t-a)\left[1-\frac{\int_{0}^{a} \frac{m(s)}{\pi(s)} \mathrm{d} s}{\left.\int_{0}^{\tau-(t-a)} \frac{m(s)}{2 \frac{5}{\pi(s)} \mathrm{d} s}\right]}\right. & \text { if } 0<t-a \leqslant \delta, \\ 0 & \text { if } \delta<t-a \leqslant \tau,\end{cases}
$$


so that we have $p(\tau, \cdot)=0$ and the control $u$ defined by $(1.23)$ is in $L^{1}\left([0, \tau] ; L^{1}\left[0, a_{\dagger}\right]\right)$.

\section{References}

[1] A. S. ACKLEH AND K. ITO, An implicit finite difference scheme for the nonlinear sizestructured population model, Numer. Funct. Anal. Optim., 18 (1997), pp. 865-884.

[2] B. AinsebA, Exact and approximate controllability of the age and space population dynamics structured model, J. Math. Anal. Appl., 275 (2002), pp. 562-574.

[3] B. Ainseba And S. Aniţa, Internal exact controllability of the linear population dynamics with diffusion, Electron. J. Differential Equations, (2004), pp. No. 112, 11 pp. (electronic).

[4] B. Ainseba And M. Iannelli, Optimal screening in structured SIR epidemics, Math. Model. Nat. Phenom., 7 (2012), pp. 12-27.

[5] B. Ainseba and M. Langlais, On a population dynamics control problem with age dependence and spatial structure, J. Math. Anal. Appl., 248 (2000), pp. 455-474.

[6] S. AniţA, Analysis and control of age-dependent population dynamics, vol. 11 of Mathematical Modelling: Theory and Applications, Kluwer Academic Publishers, Dordrecht, 2000.

[7] H. T. BANKS AND F. KAPPEL, Transformation semigroups and $L^{1}$-approximation for size structured population models, Semigroup Forum, 38 (1989), pp. 141-155. Semigroups and differential operators (Oberwolfach, 1988).

[8] V. Barbu, M. IAnnelli, and M. Martcheva, On the controllability of the LotkaMcKendrick model of population dynamics, J. Math. Anal. Appl., 253 (2001), pp. 142-165.

[9] C. FABre, Exact boundary controllability of the wave equation as the limit of internal controllability, SIAM J. Control Optim., 30 (1992), pp. 1066-1086.

[10] C. Fabre And J.-P. Puel, Behavior near the boundary for solutions of the wave equation, J. Differential Equations, 106 (1993), pp. 186-213.

[11] _ Pointwise controllability as limit of internal controllability for the wave equation in one space dimension, Portugal. Math., 51 (1994), pp. 335-350.

[12] G. H. Hardy, J. E. Littlewood, and G. PÓlya, Inequalities, Cambridge Mathematical Library, Cambridge University Press, Cambridge, 1988. Reprint of the 1952 edition.

[13] M. IAnnelLI, Mathematical theory of age-structured population dynamics, Giardini Editori e Stampatori in Pisa, 1995.

[14] K. Ito, F. Kappel, and G. Peichl, A fully discretized approximation scheme for sizestructured population models, SIAM J. Numer. Anal., 28 (1991), pp. 923-954.

[15] R. Joly, Convergence of the wave equation damped on the interior to the one damped on the boundary, J. Differential Equations, 229 (2006), pp. 588-653.

[16] F. KAPPEL AND K. P. ZhANG, Approximation of linear age-structured population models using Legendre polynomials, J. Math. Anal. Appl., 180 (1993), pp. 518-549.

[17] O. Kavian and O. Traoré, Approximate controllability by birth control for a nonlinear population dynamics model, ESAIM Control Optim. Calc. Var., 17 (2011), pp. 1198-1213. 
[18] K. Kunisch, W. Schappacher, and G. F. Webb, Nonlinear age-dependent population dynamics with random diffusion, Comput. Math. Appl., 11 (1985), pp. 155-173. Hyperbolic partial differential equations, II.

[19] P. Magal And S. Ruan, On integrated semigroups and age structured models in $L^{p}$ spaces, Differential Integral Equations, 20 (2007), pp. 197-239.

[20] — On semilinear Cauchy problems with non-dense domain, Adv. Differential Equations, 14 (2009), pp. 1041-1084.

[21] — Theory and Applications of Abstract Semilinear Cauchy Problems, Springer-Verlag New York, Cambridge University Press, Cambridge, In preparation 2017.

[22] J. Song And J. Y. Yu, Population system control, Springer-Verlag, Berlin; China Academic Publishers, Beijing, 1988. Translated from the Chinese.

[23] J. Song, J. Y. Yu, X. Z. Wang, S. J. Hu, Z. X. ZhaO, J. Q. Liu, D. X. Feng, and G. T. ZHU, Spectral properties of population operator and asymptotic behaviour of population semigroup, Acta Math. Sci. (English Ed.), 2 (1982), pp. 139-148.

[24] O. Traore, Null controllability of a nonlinear population dynamics problem, Int. J. Math. Math. Sci., (2006), pp. Art. ID 49279, 20.

[25] M. Tucsnak And G. Weiss, Observation and control for operator semigroups, Birkhäuser Advanced Texts: Basler Lehrbücher. [Birkhäuser Advanced Texts: Basel Textbooks], Birkhäuser Verlag, Basel, 2009.

[26] G. F. WeBB, Theory of nonlinear age-dependent population dynamics, vol. 89 of Monographs and Textbooks in Pure and Applied Mathematics, Marcel Dekker, Inc., New York, 1985.

[27] J. Y. Yu, B. Z. Guo, AND G. T. ZHu, Asymptotic expansion in $L\left[0, r_{m}\right]$ for population evolution and controllability of population systems, J. Systems Sci. Math. Sci., 7 (1987), pp. 97104. 